\title{
Selecting Essential Factors for Predicting Reference Crop Evapotranspiration Through Tree-based Machine Learning and Bayesian Optimisation
}

\section{Long Zhao}

Henan University of Science and Technology

\section{Xinbo Zhao}

Henan University of Science and Technology

\section{Yi Shi}

Henan University of Science and Technology

\section{Yuhang Wang}

Henan University of Science and Technology

Ningbo Cui ( $\nabla$ cuiningbo@126.com )

State Key Laboratory of Hydraulics and Mountain River Engineering \& College of Water Resource and Hydropower, Sichuan University

\section{Shuo Zhang}

Henan University of Science and Technology

\section{Research Article}

Keywords: Reference crop evapotranspiration, Essential factors, Tree-based algorithms, Machine learning, Bayesian optimization

Posted Date: December 16th, 2021

DOI: https://doi.org/10.21203/rs.3.rs-1133099/v1

License: (a) (1) This work is licensed under a Creative Commons Attribution 4.0 International License. Read Full License 


\section{Selecting essential factors for predicting reference crop evapotranspiration through tree-} based machine learning and Bayesian optimisation

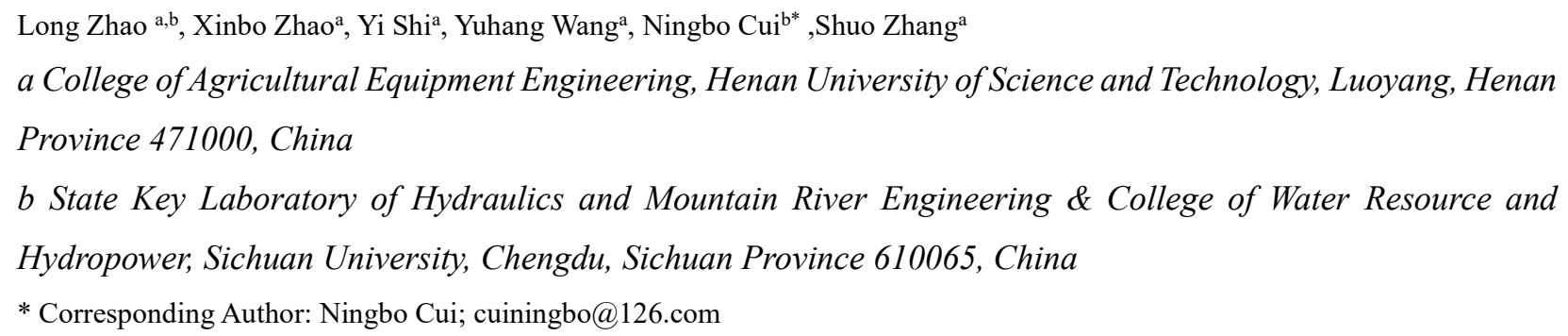

Abstract: Reference crop evapotranspiration $\left(\mathrm{ET}_{\mathrm{O}}\right)$ is a basic component of the hydrological cycle ,and its estimation is critical for agricultural water resource management and scheduling. In this study, three tree-based machine learning algorithms (random forest [RF], gradient boosting decision tree [GBDT], and extreme gradient boosting [XGBoost]) were adopted to determine the essential factors for $\mathrm{ET}_{\mathrm{O}}$ prediction. The tree-based models were optimised using the Bayesian optimisation (BO) algorithm, and they were compared with three standalone models in terms of daily $\mathrm{ET}_{\mathrm{O}}$ and monthly mean $\mathrm{ET}_{\mathrm{O}}$ estimation in North China, with different input combinations of essential variables. The results indicated that solar radiation $\left(R_{s}\right)$ and air temperature $\left(T_{s}\right)$, including the maximum, minimum, and average temperature, in daily ETo were the key parameters affecting model prediction accuracy. $\mathrm{R}_{\mathrm{s}}$ was the most influential factor in the monthly average $\mathrm{ET}_{\mathrm{O}}$ model, followed by $\mathrm{T}_{\mathrm{s}}$. Both relative humidity $(\mathrm{RH})$ and wind speed at $2 \mathrm{~m}\left(\mathrm{U}_{2}\right)$ had little impact on $\mathrm{ET}_{\mathrm{O}}$ prediction at different scales, although their importance differed. Compared with the GBDT and RF models, the XGBoost model exhibited the highest performance for daily ET $_{O}$ and monthly mean $\mathrm{ET}_{\mathrm{O}}$ estimation. The hybrid tree-based models with the $\mathrm{BO}$ algorithm outperformed the standalone tree-based models. Overall, compared with other inputs, the model with three inputs $\left(\mathrm{R}_{\mathrm{s}}, \mathrm{T}_{\mathrm{s}}\right.$, and $\left.\mathrm{RH} / \mathrm{U}_{2}\right)$ had the highest accuracy. The BO-XGBoost model exhibited superior performance in terms of the global performance index (GPI) for daily $\mathrm{ET}_{\mathrm{O}}$ and monthly mean $\mathrm{ET}_{\mathrm{O}}$ 
27 prediction and it is recommended as a more accurate model predicting daily ET $_{\mathrm{O}}$ and monthly mean

28 ETO $_{\mathrm{O}}$ in North China or areas with a similar climate.

29 Keywords: Reference crop evapotranspiration; Essential factors; Tree-based algorithms; Machine 30 learning; Bayesian optimization

\section{Introduction}

Reference crop evapotranspiration $\left(\mathrm{ET}_{\mathrm{O}}\right)$ is the core indicator for crop water resource management and water-saving irrigation planning (Hai et al., 2018; Yu et al., 2020; Zhao et al., 2020). The accurate estimation of ETo is critical for the efficient utilisation of water resources and the implementation of precision agriculture.

Many methods have been applied for measuring ETo, with the experimental measurement method being the most scientific. However, this method is challenging due to its complex experimental operations and expensive materials. Therefore, various low-cost mathematical methods for predicting $\mathrm{ET}_{\mathrm{O}}$ have been proposed, such as the United Nations Food and Agriculture Organization Penman-Monteith (FAO-56 PM; Pereira et al., 2015; Zhao et al., 2021), Hargreaves (HG), JensenHaise, and Imark-Allen methods. Among them, the FAO-56 PM method has been widely used given its wide applicability and high accuracy. However, this method requires many meteorological input factors, $T_{s}, R_{s}, R H$, and $U_{2}$ (Wang et al., 2020); these comprehensive meteorological data are often not easily obtainable, which limits the practical applications of this method (Abdullah et al., 2015; Fan et al., 2018; Wang et al., 2020; Wu et al., 2019). Few-factor ETo estimation methods, such as the Hargreaves-Samani and Priestley-Taylor models, have also been widely employed, but they cannot respond to complicated processes or be effectively optimised (Fan et al., 2018; Saeid et al., 2017; Wu et al., 2019). Therefore, the construction of a few-factor ETo model is a key research objective. Evapotranspiration is a nonlinear regression process involving various 
meteorological factors. Machine learning has obvious advantages for managing complex nonlinear

51 data and has the potential to estimate $\mathrm{ET}_{\mathrm{O}}$ (Feng et al., 2016; Yin et al., 2017). Wu and Fan (2019)

52 estimated $\mathrm{ET}_{\mathrm{O}}$ using several machine learning algorithms (i.e., support vector machine [SVM],

53 M5Tree, and extreme gradient boosting [XGBoost]) and models to achieve reliable results when

54 only temperature data was used as input. Abdullah et al. (2015) compared the prediction

55 capabilities of the elaboration likelihood model (ELM) model with that of the FAO-56 PM and

56 FFBP model and determined the ELM algorithm to have higher accuracy, faster speed, and greater

57 generalisability; thus, this model is suitable for use in places with similar climatic conditions to

58 Iraq. Feng et al. (2016) examined the accuracies of the temperature-based ELM and generalised

59 regression network neural network model (GRNN) models for estimating ET $_{O}$ in the Sichuan Basin

60 in China, and they described that these two machine learning models outperformed the

61 temperature-based HG model. Thus, researchers have demonstrated that machine learning models

62 can estimate $\mathrm{ET}_{\mathrm{O}}$ with high accuracy. Among the algorithms used for regression, tree-based

63 ensemble models, such as the M5Tree and RF models, have been widely applied given their

64 convenient processes and accurate results (Muhammed et al., 2017). Armin et al. (2014) used the

65 M5Tree model and artificial neural network, with the surface temperature of five weather stations

66 in south-eastern Iran as input data, and they reported the model's estimation as remarkable. Feng

67 et al. (2017) documented that the RF model outperformed the empirical Iqbal model in predicting

68 daily diffuse $R_{s}$ in North China. Shiri (2018) combined the RF model with the wavelet algorithm;

69 when calculating $\mathrm{ET}_{\mathrm{O}}$ in southern Iran, the performance of the RF model was markedly higher than

70 that of traditional and empirical models. Zhang et al. (2020) compared the precision of the CatBoost

71 model with that of the GRNN model tested and trained using meteorological data from 1996 to

72 2015, including daily maximum temperature $\left(\mathrm{T}_{\max }\right)$, daily minimum temperature $\left(\mathrm{T}_{\min }\right)$, wind speed 
at $2 \mathrm{~m}\left(\mathrm{U}_{2}\right), \mathrm{RH}$, and net radiation $\left(\mathrm{R}_{\mathrm{n}}\right)$; they concluded that CatBoost was more effective than the GRNN model. Wu et al. (2019) proposed various machine learning models to predict ETo using only local or cross-site temperature data and observed that tree models had higher estimation accuracy than other machine learning models. Overall, tree-based machine learning algorithms have exhibited strong performance in ETo model construction research.

However, machine learning models have a high computation time and require numerous input parameters for data training. Most of the input parameters of the tree-based models in the aforementioned studies were selected based on experience and without a theoretical basis. The selection of effective essential principal factors for $\mathrm{ET}_{\mathrm{O}}$ model construction is crucial. Some scholars have adopted principal component analysis to simplify multiple variables into a few essential variables. Through path analysis, Liu et al. (2017) identified essential meteorological variables for neural network estimation models in Wuhan and Guangzhou to simplify parameters, which allowed for a broad model application range and value. Muhammad et al. (2017) verified that the dimensionality of data was reduced through principal component analysis. The accuracy of $\mathrm{ET}_{\mathrm{O}}$ prediction reached $83 \%$ without lacking key parameters, which is more accurate than $\mathrm{ET}_{\mathrm{O}}$ prediction using other models. Although the use of the principal component analysis method to extract the main input factors is both scientific and feasible, the aforementioned study had certain regional limitations. Moreover, this method is mainly used for linear problems and is not ideal for solving nonlinear problems. Machine learning can solve nonlinear problems more effectively, and the use of the tree model to select the main factors is thus more suitable. To improve the prediction accuracy of SVR in the modelling of daily ETo, Babak and Saeid (2020) applied RF and principal component preprocessing to recognise the optimal inputs, concluding that the RF process generated more accurate results than other approaches. 
To construct a high-accuracy ETo model, we selected 12 stations in North China for ETo

97 prediction using a tree-based model. The study objectives were as follows: (1) use RF, GBDT, and

98 XGBoost to select essential factors for daily $\mathrm{ET}_{\mathrm{O}}$ and monthly mean $\mathrm{ET}_{\mathrm{O}}$ prediction; (2) compare

99 the RF, GBDT, XGBoost, and hybrid tree-based (Bayesian optimisation [BO]-RF, BO-GBDT, and

100 BO-XGBoost) models in terms of predicting daily $\mathrm{ET}_{\mathrm{O}}$ and monthly mean $\mathrm{ET}_{\mathrm{O}}$ with the essential

101 factors; and (3) evaluate all tree-based models and identify the model with the most effective

102 performance in North China on different time scales.

103 2. Materials and methods

$104 \quad 2.1$ Study area 


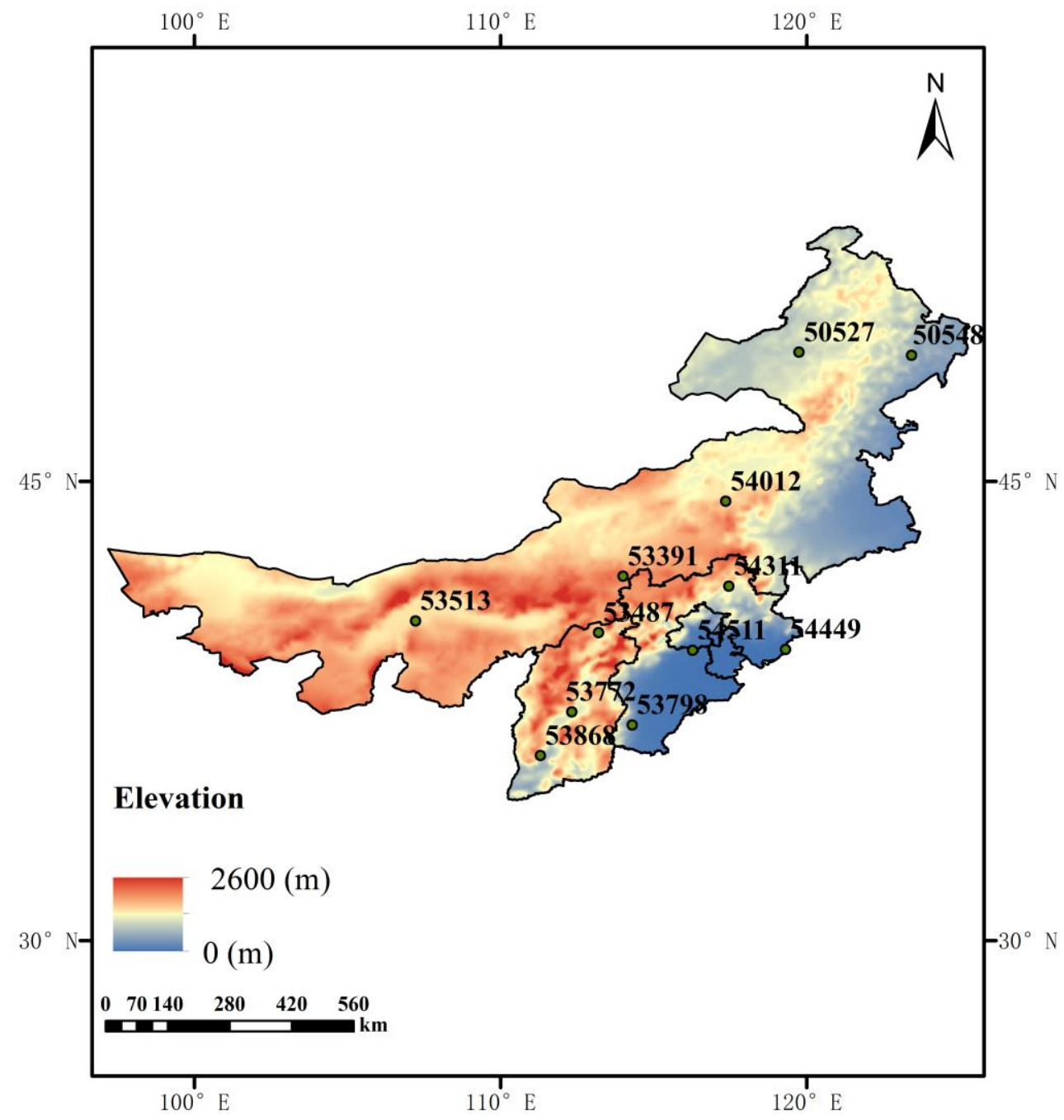

Fig. 1. Geographic distribution of the meteorological stations in North China. 
$500-600 \mathrm{~mm}$ in the area around Beijing and Tianjin.

115 The meteorological data from 1960 to 2019 were obtained from China Meteorological Data

116 Network (http://data.cma.cn). Table 1 is a detailed description of weather stations. The data were

117 divided into the training set $(80 \%)$ and test set $(20 \%)$; to reduce mistakes and improve the 118 generalisability of the model, a 10-fold cross-validation method was adopted (Fig. 2).

119 Table 1

120 Geographical locations and mean daily values of the meteorological data of the 12 weather stations

121 in this study.

\begin{tabular}{|c|c|c|c|c|c|c|c|c|c|c|}
\hline $\begin{array}{l}\text { Station } \\
\text { ID }\end{array}$ & Station name & $\begin{array}{l}\text { Latitude } \\
\left({ }^{\circ} \mathrm{N}\right)\end{array}$ & $\begin{array}{l}\text { Longitude } \\
\left({ }^{\circ} \mathrm{E}\right)\end{array}$ & $\begin{array}{l}\text { Elevation } \\
(\mathrm{m})\end{array}$ & $\begin{array}{l}\mathrm{T}_{\max } \\
\left({ }^{\circ} \mathrm{C}\right)\end{array}$ & $\begin{array}{l}\mathrm{T}_{\min } \\
\left({ }^{\circ} \mathrm{C}\right)\end{array}$ & $\begin{array}{l}\mathrm{RH} \\
(\%)\end{array}$ & $\begin{array}{l}\mathrm{U}_{2} \\
(\mathrm{~m} \\
\left.\mathrm{s}^{-1}\right)\end{array}$ & $\begin{array}{l}\mathrm{n} \\
(\mathrm{h} \\
\mathrm{d}^{-} \\
\left.{ }^{1}\right)\end{array}$ & $\begin{array}{l}\mathrm{ET}_{\mathrm{O}} \\
(\mathrm{mm} \\
\left.\mathrm{d}^{-1}\right)\end{array}$ \\
\hline 50527 & Hailaer & 49.22 & 119.75 & 139.3 & 5.3 & -6.5 & 0.6 & 2.3 & 7.2 & 2.0 \\
\hline 50548 & Xiaoergou & 49.12 & 123.43 & 139.3 & 8.3 & -7.3 & 0.7 & 1.2 & 7.2 & 1.8 \\
\hline 53391 & Huade & 41.90 & 114.00 & 286.1 & 9.5 & -2.6 & 0.6 & 3.0 & 8.2 & 2.6 \\
\hline 53487 & Datong & 40.06 & 113.20 & 1039.3 & 14.1 & 0.7 & 0.5 & 2.0 & 7.4 & 2.8 \\
\hline 53513 & Linhe & 40.44 & 107.22 & 778.3 & 14.9 & 2.0 & 0.5 & 1.7 & 8.7 & 2.9 \\
\hline 53772 & Taiyuan & 37.47 & 112.33 & 76.8 & 17.3 & 4.3 & 0.6 & 1.5 & 6.9 & 2.8 \\
\hline 53798 & Xingtai & 37.04 & 114.30 & 376.0 & 19.7 & 9.1 & 0.6 & 1.4 & 6.4 & 2.9 \\
\hline 53868 & Linfen & 36.04 & 111.30 & 995.9 & 19.3 & 7.5 & 0.6 & 1.2 & 5.9 & 2.7 \\
\hline 54012 & WestUjimqin & 44.35 & 117.36 & 842.8 & 8.7 & -4.6 & 0.6 & 2.7 & 7.8 & 2.4 \\
\hline 54311 & Weichang & 41.58 & 117.46 & 2.1 & 12.3 & -0.6 & 0.6 & 1.4 & 7.5 & 2.3 \\
\hline 54449 & Qinhuangdao & 39.51 & 119.31 & 54.0 & 15.7 & 6.4 & 0.6 & 1.8 & 7.1 & 2.6 \\
\hline 54511 & Beijing & 39.48 & 116.28 & 54.0 & 18.2 & 7.5 & 0.6 & 1.8 & 7.2 & 3.0 \\
\hline
\end{tabular}

123 temperature $\left(T_{m i n}\right)$, average relative humidity $(R H)$, sunshine hours $(n)$, and average wind speed 124 at $2 m\left(U_{2}\right)$.

\subsection{FAO-56 PM equation}

127 In this study, we used the FAO-56 PM equation ((Mirza and Kyung, 2018), 2018)as the target 128 output of the machine learning model in model training and testing, as proposed by Allen et al. 129 (1998). 


$$
E T_{0}=\frac{0.408 \Delta\left(R_{n}-G\right)+\gamma \frac{900}{T+273} U_{2}\left(e_{s}-e_{a}\right)}{\Delta+\gamma\left(1+0.34 U_{2}\right)}
$$

131 where $\Delta$ is the slope of the saturation vapour pressure temperature curve $\left(\mathrm{kPa}^{\circ} \mathrm{C}^{-1}\right) ; R_{n}$ is the net 132 solar radiation at the crop surface $\left(\mathrm{MJ} \mathrm{m}^{-2} \mathrm{~d}^{-1}\right) ; G$ is the soil heat flux density $\left(\mathrm{MJ} \mathrm{m}^{-2} \mathrm{~d}^{-1}\right) ; \gamma$ is the

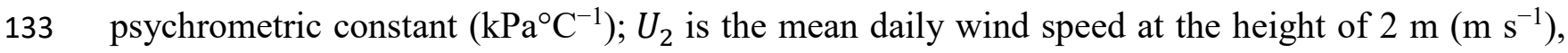
134 which is calculated from the logarithmic wind speed profile at $10 \mathrm{~m} ; e_{s}$ is the saturation vapour 135 pressure $(\mathrm{kPa}) ; e_{a}$ is the actual vapour pressure $(\mathrm{kPa})$; and $T$ is the mean air temperature at the 136 height of $2 \mathrm{~m}\left({ }^{\circ} \mathrm{C}\right)$.

137 The angstrom formula was applied to calculate $\mathrm{R}_{\mathrm{s}}$.

$$
R_{s}=\left(a_{s}+b_{s} \frac{n}{N}\right) R_{a}
$$

139 where $R_{S}$ is the solar radiation $\left(\mathrm{MJ} \mathrm{m}^{-2} \mathrm{day}^{-1}\right) ; n$ is the actual sunshine duration (h); $N$ is the 140 maximum possible sunshine duration (h); $n / N$ is the relative sunshine duration; and $R_{a}$ is the 141 zenith radiation $\left(\mathrm{MJ} \mathrm{m}^{-2} \mathrm{day}^{-1}\right)$, which also acts as the regression constant. The part of zenith 142 radiation that reaches the ground in cloudy weather is $(\mathrm{n}=0)$, with $a_{s}+b_{s}$ representing the part of 143 zenith radiation reaching the ground on a sunny day $(n=N)$; in this paper, the parameter values 144 indicated in the FAO-56 PM are $a_{s}=0.25, b_{s}=0.50$.

\section{$145 \quad 2.3$ Data processing}

146 Prior to the input processing of the importance ranking of the input data features, the data must be 147 normalised. In this study, min-max normalisation was used.

$$
x^{\prime}=\frac{x-\min (x)}{\max (x)-\min (x)}
$$

149 where $x$ and $x^{\prime}$ represent the original and normalised data, respectively. 
RF is an algorithm that was introduced by Breiman (2001). To ensure the effectiveness of the decision tree, multiple bootstrap sampling is employed to obtain numerous random samples, with 154 hundreds of decision trees forming a random forest. The nodes of each decision tree are randomly 155 generated, and the partition variables of each node are created through the random selection of a 156 few variables.

157 Because RF can assess the relationship between covariates and dependent variables and calculate the relative importance of covariates, this model can be used for feature ordering and selection. In this study, the impurity reduction method was applied to calculate feature importance. The importance of a feature can be measured based on the extent of the reduction in impurity in all trees in the forest that this feature induces. The score for each feature is calculated after training, and the results are standardised, resulting in a summed importance of all features that is equal to 1. The following Gini index calculation method was also applied:

$$
\operatorname{Gini}(\mathrm{p})=\sum_{k=1}^{K} p_{k}\left(1-p_{k}\right)
$$

where $\mathrm{k}$ represents the $\mathrm{k}$ categories, and $p_{k}$ represents the sample weight of category $\mathrm{k}$.

166 The importance on node $m$ is the change in the Gini index before and after the branch of node $m$, 167 which can be expressed as

$$
\mathrm{VIM}_{\mathrm{jm}}^{(\mathrm{Gini})}=\mathrm{GI}_{\mathrm{m}}-\mathrm{GI}_{\mathrm{l}}-\mathrm{GI}_{\mathrm{r}}
$$
where $G I_{l}$ and $G I_{r}$ represent the Gini index of the two new nodes after branching, respectively. of $X_{j}$ in the $i$-th tree is as follows:

$$
V I M_{i j}^{(G i n i)}=\sum_{m \in M} V I M_{j m}^{(G i n i)}
$$


174 by

$$
\operatorname{VIM}_{\mathrm{j}}^{(\text {Gini) }}=\sum_{\mathrm{i}=1}^{\mathrm{n}} \operatorname{VIM}_{\mathrm{ij}}^{(\text {Gini })}
$$

For regression problems, the final result of RF calculation is the average of all tree results. The regression process is described as follows:

178 First, $\mathrm{k}$ training samples $\left(\theta 1, \theta 2, \cdots \theta_{k}\right)$ are randomly generated from the total training sample using the bootstrap sampling method, corresponding to $\mathrm{K}$ decision trees that can be constructed.

At each node of the decision tree, $m$ features are randomly selected from $M$ features as the splitting feature set of the current nodes. When one node is selected from $\mathrm{m}$ features for splitting the feature set according to the principle of minimum node purity, each decision tree is grown to 183 the largest extent possible, with no pruning.

184 For new data, the predictive value of a single decision tree can be obtained by averaging the 185 observations of the leaf node $1(x, \theta)$. If an observation value $X i$ is a leaf node $1(x, \theta)$ and not 0 , 186 the weight $\omega \mathrm{i}(x, \theta)$ is set as follows:

$$
\omega_{i}(x \Theta)=\frac{1\left\{x_{i} \in R_{l}(x, \theta)\right\}}{\#\left\{j: x_{j} \in R_{l}(x, \theta)\right\}}
$$

188 where the sum of weights equals 1.

189 The prediction of a single decision tree obtained through the weighted average of the 190 observations of dependent variables is defined as follows:

$$
\mu(\mathrm{x})=\sum_{i=1}^{n} \omega_{i}(x \theta) Y_{i}
$$

where $Y_{i}(\mathrm{i}=1,2, \ldots, \mathrm{n})$ is the observation of the dependent variables. 


$$
\omega_{\mathrm{i}}(\mathrm{x})=\frac{1}{k} \sum_{i=1}^{k} \omega_{i}\left(x \Theta_{t}\right) Y
$$

Thus, the final predicted value of RFR is

$$
\mu(\mathrm{x})=\sum_{i=1}^{n} \omega_{i}(x) \mathrm{Y}_{i}
$$

\subsubsection{GBDT}

GBDT is a representative algorithm in the boosting series that is widely used in classification and regression (Fan et al., 2018). We used the squared error to represent the loss function, through which each regression tree learns the conclusions and residuals of all previous trees, which are fit to obtain the current residual regression tree. The residual is equal to the real value subtracted by the predicted value, and the lifting tree is the accumulation of the regression tree generated through the whole iterative process.

Taking the hypothetical training set sample $T=\left(x, y_{1}\right),\left(x, y_{2}\right), \ldots,\left(x, y_{m}\right)$, the maximum number of iterations is $T$, loss function is $L$, and output is a strong learner $f(x)$. The regression algorithm process is as follows:

The weak learner is initialised, and the mean value of $C$ can be set to the mean value of sample $y$.

$$
f_{0}(x)=\underbrace{\operatorname{argmin}}_{c} \sum_{i=1}^{m} L\left(y_{i}, c\right)
$$

The number of iterations $t=1,2,3, \ldots, T$ for sample $\mathrm{i}=1,2,3, \ldots, m$ is used to calculate the negative gradient as follows:

$$
r_{t i}=-\left[\frac{\partial L\left(y_{i}, f\left(x_{i}\right)\right)}{\partial f\left(x_{i}\right)}\right]_{f(x)=f_{t-1}(x)}
$$

$\left(x_{i}, r_{t i}\right) i=1,2,3, \ldots, m$ is used to fit a classification and regression tree (CART) to obtain the $t$ th regression tree. The corresponding leaf node area is $R_{t j}, j=1,2,3, \ldots, J$, where $J$ is the number of leaf nodes of regression tree $t$. 

235 becomes

236

For the leaf area $j=1,2,3, \ldots, J$, the best-fit value is calculated as follows:

$$
c_{t j}=\underbrace{\operatorname{argmin}}_{c} \sum_{x_{i} \in R_{t j}} L\left(y_{i}, f_{t-1}\left(x_{i}\right)+c\right)
$$

The strong learner $f(x)$ is updated,

$$
f_{t}(x)=f_{t-1}(x)+\sum_{j=1}^{J} c_{t j}, I\left(x \in R_{t j}\right)
$$

and the $f(x)$ expression is obtained as follows:

$$
f(x)=f_{T}(x)=f_{0}(x)+\sum_{t=1}^{T} \cdot \sum_{j=1}^{J} c_{t j}, I\left(x \in R_{t j}\right)
$$

\subsubsection{XGBoost}

XGBoost is a regression-oriented algorithm proposed by Chen and Guestrin (2016). The algorithm uses much fewer resources than other systems to expand billions of examples. In the training process, parallel computing is automatically realised. Compared with other factors, the relative importance of input factors is determined, and the built-in cross-validation model assists in preventing model overfitting, which can provide a more reliable explanation for the results. XGBoost integrates multiple regression tree models to form a strong classifier, thereby increasing the training speed, parallel processing, and generalisability. Furthermore, with more data, the parallel efficiency is higher.

XGBoost can be regarded as an additive model composed of K CARTs.

$$
\hat{y}_{i}=\sum_{k=1}^{K} f_{k}\left(x_{i}\right) f_{k} \in F
$$

where $K$ is the number of trees; $F$ is all possible CARTs; and $f_{k}$ is a specific CART.

In the return process, with the parameters $\theta=\left\{f_{1}, f_{2}, \cdots, f_{k}\right\}$, the objective function of XGBoost

$$
O(\theta)=\sum_{i=1}^{N} l\left(y_{i}, \hat{y}_{i}\right)+\sum_{k=1}^{K} \Omega\left(f_{k}\right)
$$


The first part of the formula presents the loss function, and the second part is the regular term,

238 which is obtained through the addition of the regularisation terms of the $\mathrm{K}$ trees.

239

Through vector mapping for each tree, the decision tree is improved, and the regularisation term of XGBoost is obtained as follows:

$$
\Omega\left(f_{t}\right)=\gamma T+\frac{1}{2} \lambda \sum_{j=1}^{T} \omega_{j}^{2}
$$

where $\gamma$ and $\lambda$ are the penalty coefficients of the model; $T$ is the number of leaf nodes; and $\omega$ is the

243 fraction of leaves.

244 The optimisation objective function is gradually approached step by step. At step $t$, an optimised

245 CART is added on the basis of $t-1$, and the objective function becomes

$$
O(t)=\sum_{i=1}^{N}\left(y_{i}, \hat{y}^{(t)}\right) l+\sum_{i=1}^{t} \Omega\left(f_{i}\right)=\sum_{i=1}^{N} l\left(y_{i}, \hat{y}^{(t-1)}+f_{t}\left(x_{i}\right)\right)+\Omega\left(f_{t}\right)
$$

Second-order Taylor expansion of this formula is then calculated to obtain

$$
O(t)=\sum_{i=1}^{N}\left[l\left(y_{i}, \hat{y}^{(t-1)}\right)+g_{i} f_{t}\left(x_{i}\right)+\frac{1}{2} h_{i} f_{t}^{2}\left(x_{i}\right)\right]+\Omega\left(f_{t}\right)
$$

$$
\begin{aligned}
g_{i} & =\frac{\partial\left(l\left(y_{i}, \hat{y}^{(t-1)}\right)\right)}{\partial \hat{y}^{(t-1)}} \\
h_{i} & =\frac{\partial^{2}\left(l\left(y_{i}, \hat{y}^{(t-1)}\right)\right)}{\partial \hat{y}^{(t-1)}}
\end{aligned}
$$

where $g_{i}$ and $h_{i}$ are the first and second derivatives of the loss function, respectively.

\subsection{BO algorithm}

When the calculation is complex and the number of iterations is high, $\mathrm{BO}$ can be applied. BO

254 is usually used for hyperparameter determination. For an optimised objective function, the

255 posterior distribution of the objective function can be updated through the continualaddition of 256 sample points until the posterior distribution largely fits the real distribution. BO has two core 
processes, namely prior function (PF) and acquisition function (AC). PF mainly uses Gaussian 258 process regression, whereas AC includes expected improvement, the probability of improvement, 259 the upper confidence bound of these methods, and the balance of exploration and exploitation. To 260 achieve exploration, a point far away from the known point is selected as the reference point for 261 the next iteration (to explore the unknown area), with the point distribution computed as evenly as 262 possible. For exploitation, a point close to the known point is selected as the reference point for the 263 next iteration (to exploit the points around the known point), with points distributed in a dense area, 264 facilitating achievement of the local maximum.

265 If a set of hyperparameter combinations $X=x_{1}, x_{2}, \ldots, x_{n}, x_{n}$ represents the value of a 266 certain hyperparameter, BO assumes a functional relationship between the hyperparameters and 267 the loss function that requires optimisation. For example, take the function $f: X \rightarrow \mathbb{R}, X \subset$ $268 \mathbb{R}^{n}, n \in N$. In each iteration, $f\left(x_{t}\right), x_{t} \in X(t=1,2, \cdots, T)$ is obtained according to the AC. 269 Then, $y_{t}=f\left(x_{t}\right)+\varepsilon$ is obtained, with $\varepsilon$ obeying the zero mean Gaussian distribution $\varepsilon \sim$ $270 \mathcal{N}\left(0, \sigma^{2}\right) ; \sigma$ denotes the variance. The new observation data $\left(x_{t}, y_{t}\right)$ are added to the original 271 observation data, and the next iteration is then executed. Through the learning of the objective 272 function, BO uses the information of the previous sampling point to identify the parameters that 273 would improve and move the result toward the global optimum. The algorithm tests the most 274 probable point in the posterior distribution. Fig. 2 depicts the entire analytical process of the 275 optimisation model for estimating $\mathrm{ET}_{\mathrm{O}}$. 


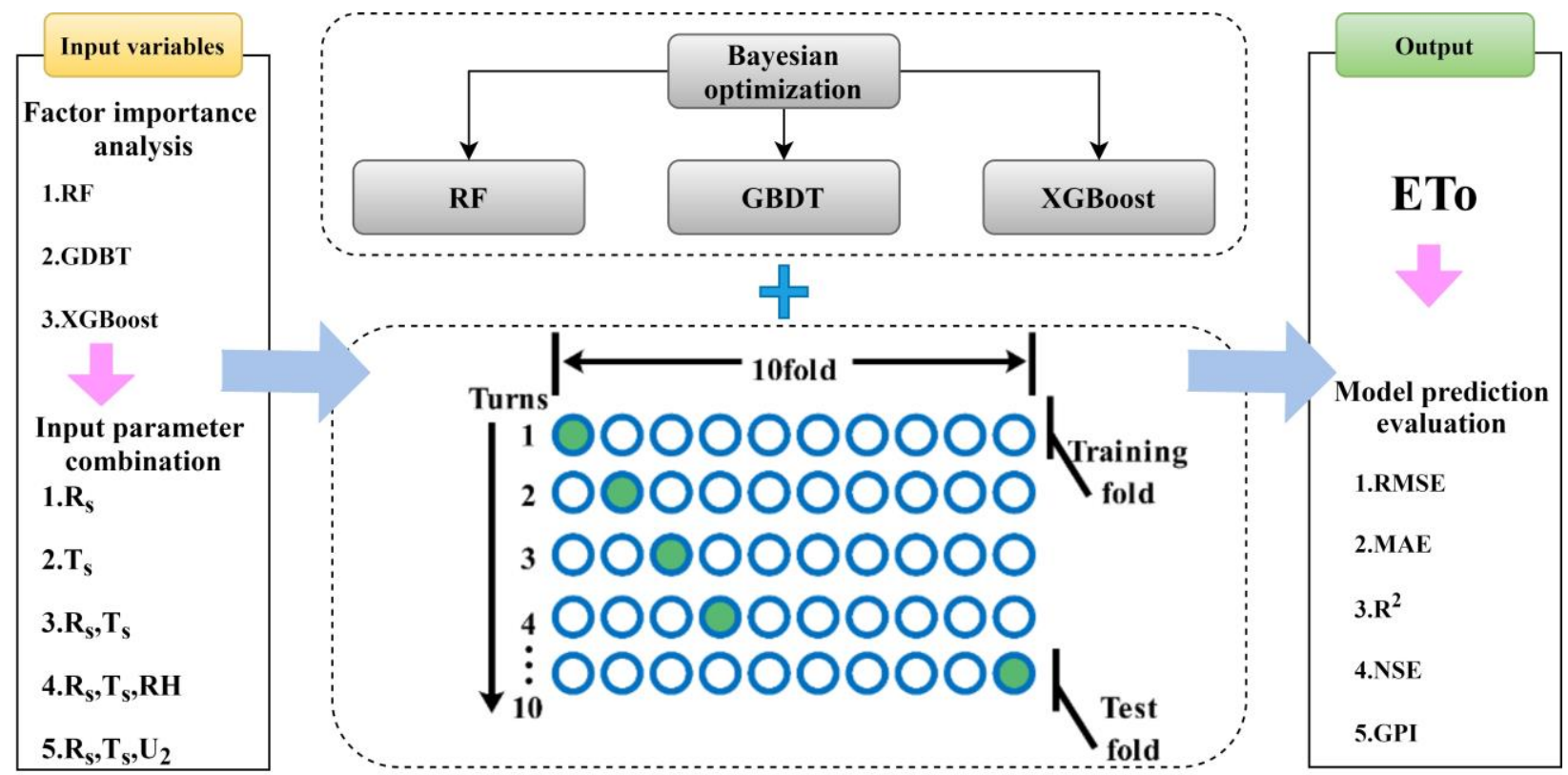

Fig. 2. Flowchart of the reference crop evapotranspiration prediction model used in this study

\subsection{Model prediction evaluation} performance of the models (Feng et al., 2015; Shiri et al., 2015).

$$
\mathrm{R}^{2}=\frac{\left[\sum_{\mathrm{i}=1}^{\mathrm{n}} \mathrm{Si}-\overline{\mathrm{S}}(\mathrm{Oi}-\overline{\mathrm{O}})\right]^{2}}{\sum_{\mathrm{i}=1}^{\mathrm{n}} \mathrm{Si}-\overline{\mathrm{s}}^{2} \sum_{\mathrm{i}=1}^{\mathrm{n}}(\mathrm{Oi}-\overline{\mathrm{O}})^{2}}
$$

$$
\mathrm{MAE}=\frac{1}{\mathrm{n}} \sum_{\mathrm{i}=1}^{\mathrm{n}}|\mathrm{Si}-\mathrm{Oi}|
$$

$$
\mathrm{GPI}=\alpha_{\mathrm{j}} \sum_{\mathrm{i}=1}^{4}\left(\mathrm{~T}_{\mathrm{j}}-\overline{\mathrm{T}}_{\mathrm{j}}\right)
$$

where $X_{i}$ and $Y_{i}$ are the simulated and measured values, respectively; $n$ is the number of measured 
values; and $\bar{x}$ and $\bar{y}$ are the means of the simulated and measured values, respectively. $T_{j}$ is the normalised value of RMSE, MAE, $\mathrm{R}^{2}$, and NSE, and $\bar{T}_{j}$ is the median of the corresponding parameter; when $T_{j}$ is RMSE and MAE, $\alpha_{j}$ is -1 or 1 otherwise. The closer $\mathrm{R}^{2}$ is to 1 , the more accurate is the prediction ability of the model, and the smaller the value of MAE and RMSE, the smaller the simulation error. The closer NSE is to 1 , the higher the model quality and credibility. The higher the GPI, the more effective the overall simulation effect of the model.

\section{Results}

\subsection{Factor importance analysis based on tree-based algorithms}

Three tree-based algorithms, namely RF, GBDT, and XGBoost, were applied to determine the essential meteorological factors for daily $\mathrm{ET}_{O}$ and monthly mean $\mathrm{ET}_{\mathrm{O}}$ prediction. Importance weights between 0 and 1 were assigned to $T_{\max }, T_{\min }, T_{\text {mean }}, R H, U_{2}$, and $R_{s}$ in these three methods, with the importance being used to determine the most effective meteorological factors on different time scales. Fig. 3 and Fig. 4 present the results of the factor importance analysis based on the treebased algorithms according to the heat map. Overall, the algorithms exhibited varying performance in the daily $\mathrm{ET}_{\mathrm{O}}$ and monthly mean $\mathrm{ET}_{\mathrm{O}}$ factor importance analysis, but the importance tended to be consistent on the same time scale.

For the daily $\mathrm{ET}_{\mathrm{O}}$ factor importance analysis (Fig. 3 and Table 2) in five selected sites in Inner Mongolia (50527, 50548, 53391, 53513, and 54012), $\mathrm{T}_{\mathrm{s}}$ was the most important influencing factor, ranging from 0.717 to $0.795,0.675$ to 0.867 , and 0.721 to 0.794 for RF, GBDT, and XGBoost, respectively, followed by $R_{s}$, ranging from 0.115 to $0.216,0.091$ to 0.250 , and 0.116 to 0.218 , respectively. Among the other sites selected in North China, $\mathrm{R}_{\mathrm{s}}$ was the most influential factor, ranging from 0.700 to $0.759,0.499$ to 0.680 , and 0.699 to 0.758 for RF, GBDT, and XGBoost, 
311 respectively, followed by $\mathrm{T}_{\mathrm{s}}$, ranging from 0.115 to $0.228,0.245$ to 0.421 , and 0.152 to 0.229 , 312 respectively. $\mathrm{RH}$ and $\mathrm{U}_{2}$ consistently ranked third and fourth in importance at all sites.

313 In the factor importance analysis of the monthly mean $\mathrm{ET}_{\mathrm{O}}, \mathrm{R}_{\mathrm{S}}$ had the most importance at all 314 study sites, ranging from 0.638 to $0.970,0.855$ to 0.967 , and 0.567 to 0.857 for RF, GBDT, and 315 XGBoost, respectively (Fig. 4 and Table 2). After $R_{s}, T_{s}$ exhibited the most importance, ranging 316 from 0.021 to $0.035,0.022$ to 0.136 , and 0.128 to 0.410 for RF, GBDT, and XGBoost, respectively, 317 followed by $\mathrm{RH}$ and $\mathrm{U}_{2}$.

Most of the importance analysis results of the three models on the daily and monthly scales were the same. Because the contribution of $\mathrm{R}_{\mathrm{s}}$ and $\mathrm{T}_{\mathrm{s}}$ differed considerably in different regions, we used $\mathrm{R}_{\mathrm{s}}$ and $\mathrm{T}_{\mathrm{s}}$ as the basic input combination, with both input at the same time. Finally, $\mathrm{RH}$ and $321 \mathrm{U}_{2}$ were introduced. (Table 3).

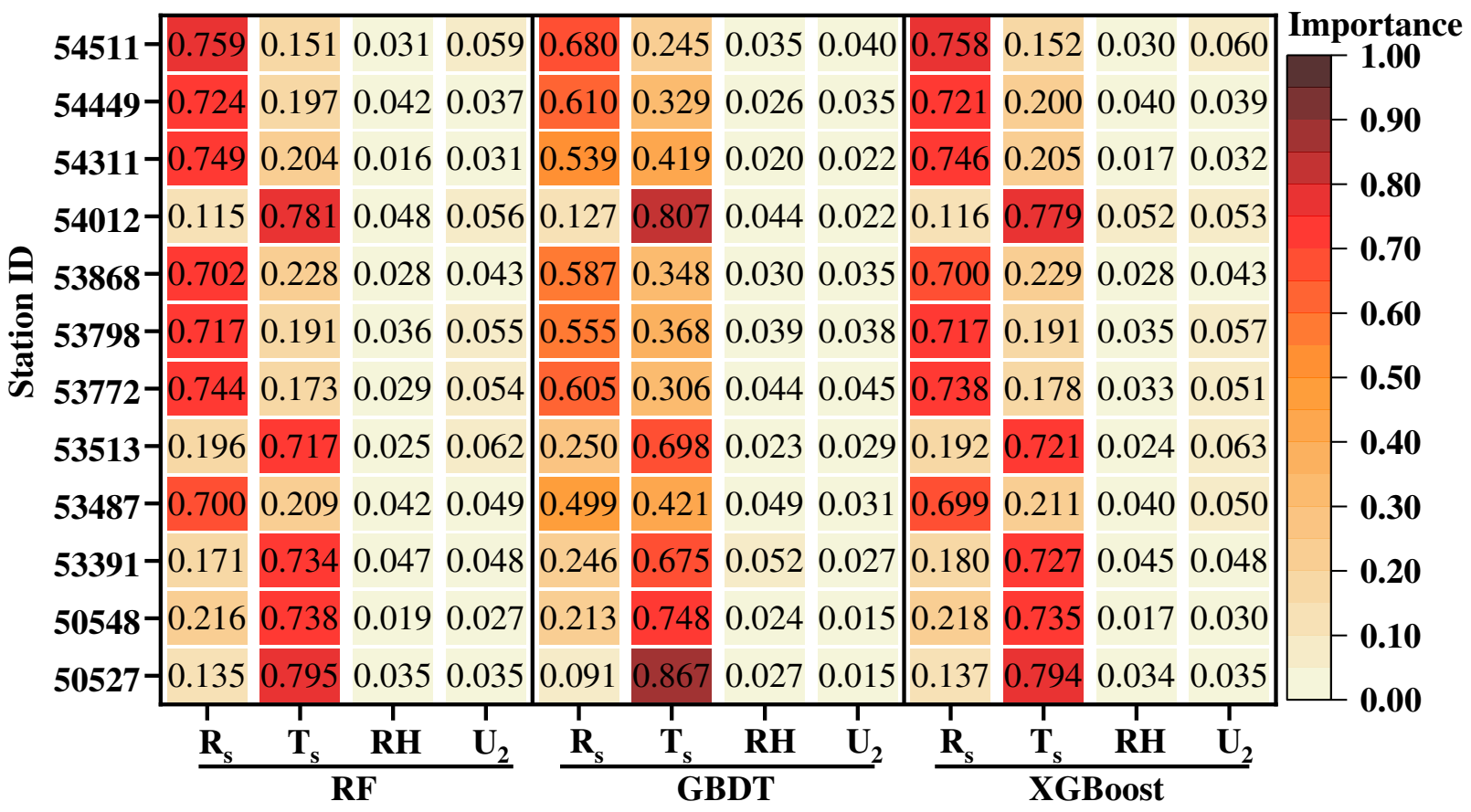

Fig. 3. Heat map of the factor importance analysis of daily $\mathrm{ET}_{\mathrm{O}}$. 


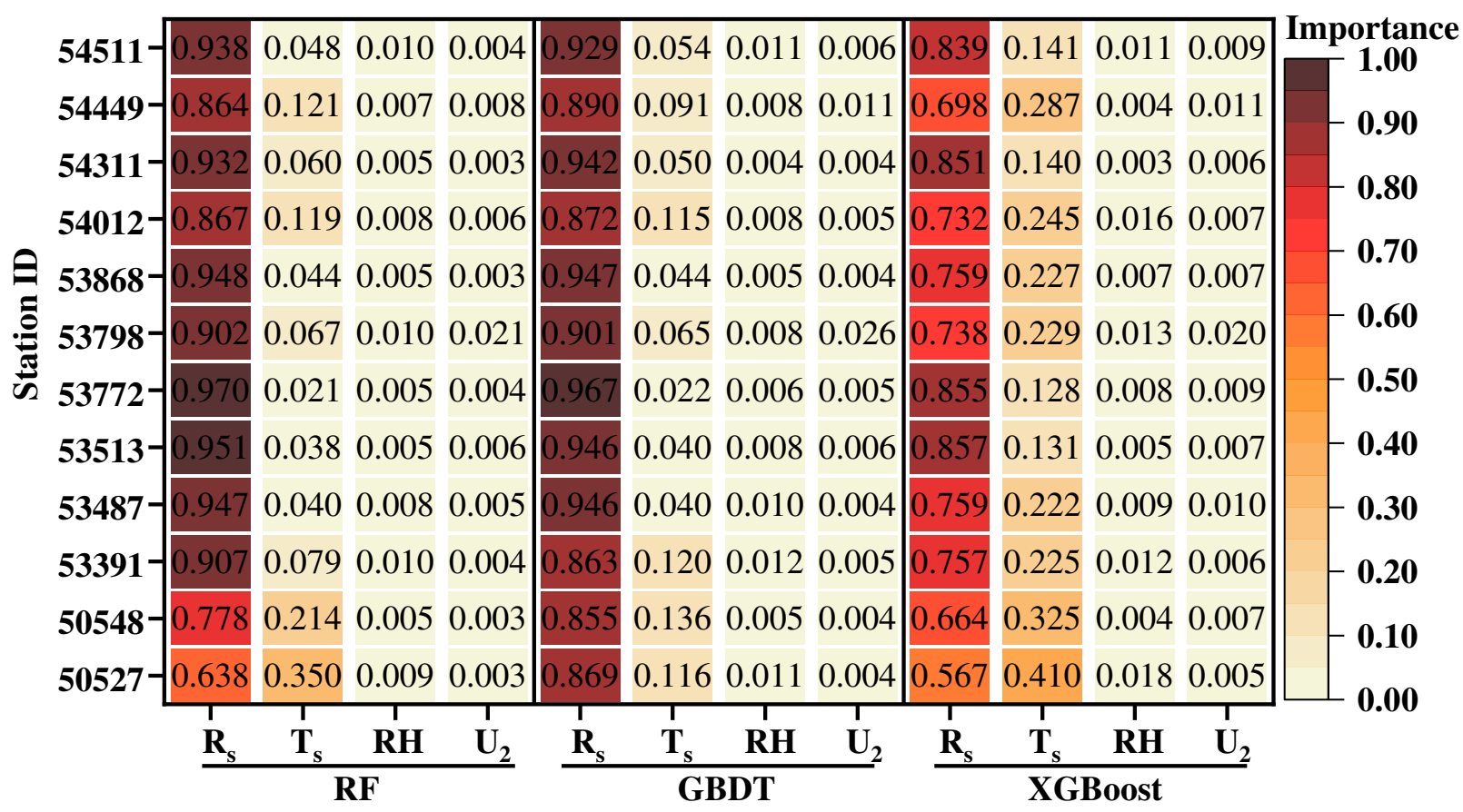

Fig. 4. Heat map of the factor importance analysis of monthly mean ETo.

328 Table 2

329 Factor importance analysis of daily $\mathrm{ET}_{\mathrm{O}}$ and monthly mean $\mathrm{ET}_{\mathrm{O}}$.

\begin{tabular}{|c|c|c|c|c|c|c|c|c|c|c|c|c|}
\hline \multicolumn{7}{|c|}{${\text { Daily } \mathbf{E T}_{0}}$} & \multicolumn{6}{|c|}{ Monthly mean ETo $_{0}$} \\
\hline Station & $\mathrm{T}_{\text {mean }}$ & $\mathrm{T}_{\max }$ & $\mathrm{T}_{\min }$ & $\mathrm{U}_{2}$ & RH & $\mathrm{R}_{\mathrm{s}}$ & $\mathrm{T}_{\text {mean }}$ & $\mathrm{T}_{\max }$ & $\mathrm{T}_{\min }$ & $\mathrm{U}_{2}$ & RH & $\mathrm{R}_{\mathrm{s}}$ \\
\hline & & & RF & & & & \multicolumn{6}{|c|}{ RF } \\
\hline 50527 & 0.279 & 0.512 & 0.004 & 0.035 & 0.036 & 0.135 & 0.046 & 0.297 & 0.007 & 0.003 & 0.009 & 0.638 \\
\hline 50548 & 0.724 & 0.009 & 0.005 & 0.027 & 0.019 & 0.216 & 0.118 & 0.083 & 0.012 & 0.003 & 0.005 & 0.778 \\
\hline 53391 & 0.032 & 0.698 & 0.004 & 0.048 & 0.047 & 0.171 & 0.007 & 0.063 & 0.009 & 0.004 & 0.010 & 0.907 \\
\hline 53487 & 0.069 & 0.136 & 0.004 & 0.049 & 0.042 & 0.700 & 0.011 & 0.022 & 0.007 & 0.005 & 0.008 & 0.947 \\
\hline 53513 & 0.518 & 0.196 & 0.003 & 0.062 & 0.025 & 0.196 & 0.012 & 0.021 & 0.005 & 0.006 & 0.005 & 0.952 \\
\hline 53772 & 0.047 & 0.122 & 0.004 & 0.054 & 0.029 & 0.744 & 0.005 & 0.013 & 0.003 & 0.004 & 0.005 & 0.970 \\
\hline 53798 & 0.037 & 0.148 & 0.006 & 0.055 & 0.036 & 0.717 & 0.017 & 0.039 & 0.012 & 0.021 & 0.010 & 0.902 \\
\hline 53868 & 0.032 & 0.192 & 0.004 & 0.043 & 0.028 & 0.702 & 0.008 & 0.033 & 0.003 & 0.003 & 0.005 & 0.948 \\
\hline 54012 & 0.710 & 0.067 & 0.004 & 0.056 & 0.048 & 0.115 & 0.021 & 0.095 & 0.004 & 0.006 & 0.008 & 0.867 \\
\hline 54311 & 0.174 & 0.023 & 0.007 & 0.031 & 0.016 & 0.749 & 0.026 & 0.019 & 0.015 & 0.003 & 0.005 & 0.932 \\
\hline 54449 & 0.120 & 0.052 & 0.025 & 0.037 & 0.042 & 0.724 & 0.034 & 0.051 & 0.035 & 0.008 & 0.007 & 0.864 \\
\hline 54511 & 0.055 & 0.075 & 0.022 & 0.059 & 0.031 & 0.759 & 0.014 & 0.023 & 0.011 & 0.004 & 0.010 & 0.938 \\
\hline \multicolumn{7}{|c|}{ GBDT } & \multicolumn{6}{|c|}{ GBDT } \\
\hline 50548 & 0.578 & 0.281 & 0.008 & 0.015 & 0.027 & 0.091 & 0.016 & 0.091 & 0.009 & 0.004 & 0.011 & 0.870 \\
\hline 53399 & 0.719 & 0.018 & 0.011 & 0.015 & 0.024 & 0.213 & 0.019 & 0.094 & 0.023 & 0.004 & 0.005 & 0.855 \\
\hline 53446 & 0.081 & 0.567 & 0.028 & 0.027 & 0.052 & 0.246 & 0.003 & 0.103 & 0.014 & 0.005 & 0.011 & 0.863 \\
\hline 53463 & 0.167 & 0.239 & 0.015 & 0.031 & 0.049 & 0.499 & 0.003 & 0.022 & 0.015 & 0.004 & 0.010 & 0.946 \\
\hline 53513 & 0.479 & 0.212 & 0.008 & 0.029 & 0.022 & 0.250 & 0.011 & 0.027 & 0.002 & 0.006 & 0.008 & 0.946 \\
\hline 53772 & 0.112 & 0.176 & 0.018 & 0.045 & 0.044 & 0.605 & 0.006 & 0.015 & 0.001 & 0.004 & 0.006 & 0.967 \\
\hline 53798 & 0.114 & 0.235 & 0.019 & 0.037 & 0.039 & 0.555 & 0.018 & 0.042 & 0.005 & 0.025 & 0.008 & 0.901 \\
\hline 53959 & 0.084 & 0.244 & 0.019 & 0.035 & 0.030 & 0.587 & 0.005 & 0.036 & 0.003 & 0.004 & 0.005 & 0.948 \\
\hline 54012 & 0.527 & 0.274 & 0.006 & 0.022 & 0.044 & 0.127 & 0.001 & 0.112 & 0.001 & 0.005 & 0.008 & 0.872 \\
\hline 54135 & 0.345 & 0.056 & 0.018 & 0.022 & 0.020 & 0.539 & 0.015 & 0.005 & 0.031 & 0.004 & 0.004 & 0.942 \\
\hline 54311 & 0.191 & 0.091 & 0.046 & 0.035 & 0.026 & 0.610 & 0.014 & 0.047 & 0.031 & 0.011 & 0.008 & 0.890 \\
\hline 54449 & 0.122 & 0.078 & 0.044 & 0.040 & 0.035 & 0.680 & 0.003 & 0.022 & 0.029 & 0.006 & 0.011 & 0.929 \\
\hline \multicolumn{7}{|c|}{ XGBoost } & \multicolumn{6}{|c|}{ XGBoost } \\
\hline 50548 & 0.015 & 0.776 & 0.003 & 0.035 & 0.034 & 0.137 & 0.020 & 0.378 & 0.012 & 0.005 & 0.018 & 0.567 \\
\hline 53399 & 0.721 & 0.011 & 0.002 & 0.030 & 0.017 & 0.218 & 0.185 & 0.135 & 0.005 & 0.007 & 0.004 & 0.664 \\
\hline 53446 & 0.012 & 0.712 & 0.003 & 0.048 & 0.045 & 0.180 & 0.024 & 0.192 & 0.010 & 0.006 & 0.012 & 0.757 \\
\hline 53463 & 0.069 & 0.139 & 0.003 & 0.050 & 0.040 & 0.699 & 0.085 & 0.127 & 0.010 & 0.010 & 0.009 & 0.759 \\
\hline 53513 & 0.657 & 0.061 & 0.003 & 0.063 & 0.024 & 0.192 & 0.023 & 0.106 & 0.002 & 0.007 & 0.005 & 0.857 \\
\hline
\end{tabular}


Table 3

331 Input combinations of the meteorological factors for various models.

\begin{tabular}{|c|c|c|c|c|c|c|}
\hline \multicolumn{6}{|c|}{ Models } & \multirow{2}{*}{ Input combinations } \\
\hline $\mathrm{RF}$ & GBDT & XGBoost & BO-RF & BO-GBDT & BO-XGBoost & \\
\hline \multicolumn{7}{|c|}{ Daily ETo predicting } \\
\hline RF1 & GBDT1 & XGBoost1 & BO-RF1 & BO-GBDT1 & BO-XGBoost1 & $\mathrm{R}_{\mathrm{s}}$ \\
\hline RF2 & GBDT2 & XGBoost2 & BO-RF2 & BO-GBDT2 & BO-XGBoost2 & $\mathrm{T}_{\mathrm{s}}$ \\
\hline $\mathrm{RF} 3$ & GBDT3 & XGBoost3 & BO-RF3 & BO-GBDT3 & BO-XGBoost3 & $\mathrm{R}_{\mathrm{s}}, \mathrm{T}_{\mathrm{s}}$ \\
\hline RF4 & GBDT4 & XGBoost4 & BO-RF4 & BO-GBDT4 & BO-XGBoost4 & $\mathrm{R}_{\mathrm{s}}, \mathrm{T}_{\mathrm{s}} \mathrm{RH}$ \\
\hline RF5 & GBDT5 & XGBoost5 & BO-RF5 & BO-GBDT5 & BO-XGBoost5 & $\mathrm{R}_{\mathrm{s}}, \mathrm{T}_{\mathrm{s}} \mathrm{U}_{2}$ \\
\hline \multicolumn{7}{|c|}{ Monthly mean ETo predicting } \\
\hline RF6 & GBDT6 & XGBoost6 & BO-RF6 & BO-GBDT6 & BO-XGBoost6 & $\mathrm{R}_{\mathrm{s}}$ \\
\hline RF7 & GBDT7 & XGBoost7 & BO-RF7 & BO-GBDT7 & BO-XGBoost7 & $\mathrm{T}_{\mathrm{s}}$ \\
\hline RF8 & GBDT8 & XGBoost8 & BO-RF8 & BO-GBDT8 & BO-XGBoost8 & $\mathrm{R}_{\mathrm{s}}, \mathrm{T}_{\mathrm{s}}$ \\
\hline RF9 & GBDT9 & XGBoost9 & BO-RF9 & BO-GBDT9 & BO-XGBoost9 & $\mathrm{R}_{\mathrm{s}}, \mathrm{T}_{\mathrm{s}} \mathrm{RH}$ \\
\hline RF10 & GBDT10 & XGBoost10 & BO-RF10 & BO-GBDT10 & BO-XGBoost10 & $\mathrm{R}_{\mathrm{s}}, \mathrm{T}_{\mathrm{s}} \mathrm{U}_{2}$ \\
\hline
\end{tabular}

332

338 and monthly mean scale under different input combinations in the testing phase are illustrated in 339 Fig. 5 and Fig. 6. The same letter denotes no significant difference between the models, as 340 determined using Fisher's least significant difference at a 0.05 significance level. 
342 between the $\mathrm{R}_{\mathrm{S}}$ and $\mathrm{T}_{\mathrm{s}}$ input $(p>0.05)$, indicating the similar overall accuracy of these two factors.

343 When the two factors were input, the difference was obvious compared with single-factor input ( $p$ $344<0.05)$. Three-factor input considerably improved the accuracy of most models; however, the RF 345 model did not exhibit a marked improvement compared with the two-factor input condition. 346 Compared with the accuracy of the single-factor model, the three-input RF model showed 347 significantly improved performance $(p<0.05)$. No significant difference was noted among the 348 three-factor combinations (with $\mathrm{RH}$ or $\left.\mathrm{U}_{2}\right)$ in terms of the model accuracy indicators $(p>0.05)$, 349 which was consistent with the main factor analysis result. As illustrated in Fig. 5, for monthly 350 average $\mathrm{ET}_{\mathrm{O}}$ estimation, a significant correlation was found between the single-factor and double351 factor input, exhibiting the same trend as that of daily $\mathrm{ET}_{\mathrm{O}}$. When two factors were input, the 352 difference was obvious compared with single-factor input $(p<0.05)$. Three-factor input 353 considerably improved the accuracy of most models, but the RF model did not show marked 354 improvement compared with the two-factor input model. However, the three-input RF model 355 showed significant improvement in terms of accuracy compared with the single-factor model ( $p<$ 356 0.05). No significant difference was noted among the three-factor combinations (with $\mathrm{RH}$ or $\mathrm{U}_{2}$ ) 357 in terms of the model accuracy indicators $(p>0.05)$ Compared with single-factor input, the 358 accuracy of the three-factor input model considerably improved, but the accuracy of the two-factor 359 input model did not exhibit significant improvement $(p>0.05)$.

As detailed in Table 4, under the different input combinations, the XGBoost models 361 outperformed the other models in the training phase, with RMSE, $\mathrm{R}^{2}, \mathrm{MAE}$, and NSE ranging from 3620.316 to $0.821 \mathrm{~mm} \mathrm{~d}^{-1}, 0.796$ to $0.969,0.220$ to $0.623 \mathrm{~mm} \mathrm{~d}^{-1}$, and 0.796 to 0.969 , respectively, 363 and from 0.335 to $0.836 \mathrm{~mm} \mathrm{~d}^{-1}, 0.789$ to $0.966,0.232$ to $0.633 \mathrm{~mm} \mathrm{~d}^{-1}$, and 0.789 to 0.966 , 

models. In addition to the significance difference between the standalone and hybrid models,

366 significant differences were observed in $\mathrm{R}^{2}$, RMSE, MAE, and NSE under different input 367 combinations, indicating that the hybrid BO algorithm significantly improved the tree-based 368 algorithms. No significant difference was noted among the three hybrid models, with all hybrid models exhibiting high accuracy compared with the standalone tree-based models. Overall, the

370 BO-XGBoost model had highest accuracy among the other tree-based models in the training

371 phaseRMSE, $\mathrm{R}^{2}, \mathrm{MAE}$, and NSE ranging from 0.263 to $0.737 \mathrm{~mm} \mathrm{~d}^{-1}, 0.834$ to $0.979,0.182$ to $3720.555 \mathrm{~mm} \mathrm{~d}^{-1}$, and 0.834 to 0.979 , respectively, and from 0.317 to $0.811 \mathrm{~mm} \mathrm{~d}^{-1}, 0.801$ to 0.969 , 3730.214 to $0.606 \mathrm{~mm} \mathrm{~d}^{-1}$, and 0.801 to 0.969 , respectively, in the testing phase; this was followed by 374 the performance of the BO-GBDT and BO-RF models.

The BO-XGBoost model had the highest accuracy along with the other tree-based models, 376 and the XGBoost models outperformed the GBDT models, followed by the RF models. The 377 accuracy of the model increased with the increased number of input parameters, which is consistent 378 with the main factor results.

\section{Table 4}

$\mathrm{R}^{2}$, RMSE, MAE, and NSE values of $\mathrm{ET}_{\mathrm{O}}$ prediction during the training and testing phases.

\begin{tabular}{|c|c|c|c|c|c|c|c|c|}
\hline \multirow{2}{*}{ Input } & \multicolumn{4}{|c|}{ Training } & \multicolumn{4}{|c|}{ Testing } \\
\hline & $\operatorname{RMSE}\left(\mathrm{mm} \mathrm{d}^{-1}\right)$ & $\mathrm{R}^{2}$ & $\operatorname{MAE}\left(\mathrm{mm} \mathrm{d}^{-1}\right)$ & NSE & $\operatorname{RMSE}\left(\mathrm{mm} \mathrm{d}^{-1}\right)$ & $\mathrm{R}^{2}$ & $\operatorname{MAE}\left(\mathrm{mm} \mathrm{d}^{-1}\right)$ & NSE \\
\hline \multicolumn{9}{|l|}{$\overline{\mathrm{R}_{\mathrm{s}}}$} \\
\hline RF1 & $0.832 \pm 0.119$ & $0.790 \pm 0.034$ & $0.633 \pm 0.076$ & $0.790 \pm 0.034$ & $0.841 \pm 0.129$ & $0.786 \pm 0.039$ & $0.638 \pm 0.081$ & $0.786 \pm 0.039$ \\
\hline GBDT1 & $0.822 \pm 0.119$ & $0.795 \pm 0.034$ & $0.624 \pm 0.077$ & $0.795 \pm 0.034$ & $0.837 \pm 0.131$ & $0.789 \pm 0.040$ & $0.634 \pm 0.083$ & $0.789 \pm 0.040$ \\
\hline XGBoost1 & $0.821 \pm 0.119$ & $0.796 \pm 0.034$ & $0.623 \pm 0.077$ & $0.796 \pm 0.034$ & $0.836 \pm 0.131$ & $0.789 \pm 0.040$ & $0.633 \pm 0.083$ & $0.789 \pm 0.040$ \\
\hline BO-RF1 & $0.824 \pm 0.120$ & $0.794 \pm 0.036$ & $0.627 \pm 0.077$ & $0.794 \pm 0.036$ & $0.840 \pm 0.129$ & $0.787 \pm 0.039$ & $0.637 \pm 0.081$ & $0.787 \pm 0.039$ \\
\hline BO-GBDT1 & $0.746 \pm 0.149$ & $0.830 \pm 0.047$ & $0.558 \pm 0.103$ & $0.830 \pm 0.047$ & $0.815 \pm 0.139$ & $0.799 \pm 0.045$ & $0.607 \pm 0.096$ & $0.799 \pm 0.045$ \\
\hline BO-XGBoost1 & $0.737 \pm 0.139$ & $0.834 \pm 0.043$ & $0.555 \pm 0.096$ & $0.834 \pm 0.043$ & $0.811 \pm 0.141$ & $0.801 \pm 0.046$ & $0.606 \pm 0.096$ & $0.801 \pm 0.046$ \\
\hline \multicolumn{9}{|l|}{$\overline{\mathrm{T}_{\mathrm{s}}}$} \\
\hline RF2 & $0.852 \pm 0.096$ & $0.775 \pm 0.053$ & $0.634 \pm 0.091$ & $0.775 \pm 0.053$ & $0.865 \pm 0.093$ & $0.769 \pm 0.054$ & $0.643 \pm 0.089$ & $0.769 \pm 0.054$ \\
\hline GBDT2 & $0.792 \pm 0.092$ & $0.806 \pm 0.044$ & $0.584 \pm 0.084$ & $0.806 \pm 0.044$ & $0.806 \pm 0.088$ & $0.800 \pm 0.042$ & $0.595 \pm 0.081$ & $0.800 \pm 0.042$ \\
\hline XGBoost2 & $0.772 \pm 0.090$ & $0.815 \pm 0.044$ & $0.568 \pm 0.083$ & $0.815 \pm 0.044$ & $0.791 \pm 0.084$ & $0.808 \pm 0.041$ & $0.582 \pm 0.080$ & $0.808 \pm 0.041$ \\
\hline BO-RF2 & $0.812 \pm 0.086$ & $0.796 \pm 0.046$ & $0.600 \pm 0.082$ & $0.796 \pm 0.046$ & $0.825 \pm 0.085$ & $0.791 \pm 0.046$ & $0.610 \pm 0.080$ & $0.791 \pm 0.046$ \\
\hline BO-GBDT2 & $0.713 \pm 0.094$ & $0.842 \pm 0.044$ & $0.527 \pm 0.085$ & $0.842 \pm 0.044$ & $0.778 \pm 0.086$ & $0.815 \pm 0.039$ & $0.572 \pm 0.080$ & $0.815 \pm 0.039$ \\
\hline
\end{tabular}




\begin{tabular}{|c|c|c|c|c|c|c|c|c|}
\hline BO-XGBoost2 & $0.709 \pm 0.081$ & $0.846 \pm 0.024$ & $0.522 \pm 0.067$ & $0.846 \pm 0.024$ & $0.745 \pm 0.072$ & $0.831 \pm 0.023$ & $0.547 \pm 0.065$ & $0.831 \pm 0.023$ \\
\hline \multicolumn{9}{|l|}{$\mathrm{R}_{\mathrm{s}}, \mathrm{T}_{\mathrm{s}}$} \\
\hline RF3 & $0.645 \pm 0.081$ & $0.874 \pm 0.018$ & $0.467 \pm 0.060$ & $0.874 \pm 0.018$ & $0.659 \pm 0.085$ & $0.869 \pm 0.019$ & $0.476 \pm 0.060$ & $0.869 \pm 0.019$ \\
\hline GBDT3 & $0.514 \pm 0.086$ & $0.919 \pm 0.017$ & $0.356 \pm 0.065$ & $0.919 \pm 0.017$ & $0.539 \pm 0.091$ & $0.912 \pm 0.019$ & $0.370 \pm 0.068$ & $0.912 \pm 0.019$ \\
\hline XGBoost3 & $0.515 \pm 0.086$ & $0.919 \pm 0.017$ & $0.356 \pm 0.066$ & $0.919 \pm 0.017$ & $0.539 \pm 0.091$ & $0.912 \pm 0.019$ & $0.370 \pm 0.068$ & $0.912 \pm 0.019$ \\
\hline BO-RF3 & $0.576 \pm 0.083$ & $0.899 \pm 0.017$ & $0.406 \pm 0.062$ & $0.899 \pm 0.017$ & $0.592 \pm 0.086$ & $0.894 \pm 0.018$ & $0.417 \pm 0.062$ & $0.894 \pm 0.018$ \\
\hline BO-GBDT3 & $0.492 \pm 0.096$ & $0.926 \pm 0.02$ & $0.340 \pm 0.071$ & $0.926 \pm 0.02$ & $0.530 \pm 0.093$ & $0.915 \pm 0.019$ & $0.363 \pm 0.069$ & $0.915 \pm 0.019$ \\
\hline BO-XGBoost3 & $0.473 \pm 0.094$ & $0.931 \pm 0.02$ & $0.327 \pm 0.068$ & $0.931 \pm 0.02$ & $0.529 \pm 0.093$ & $0.915 \pm 0.019$ & $0.360 \pm 0.069$ & $0.915 \pm 0.019$ \\
\hline \multicolumn{9}{|l|}{$\overline{\mathrm{R}_{\mathrm{s}}, \mathrm{T}_{\mathrm{s}}, \mathrm{RH}}$} \\
\hline RF4 & $0.642 \pm 0.077$ & $0.875 \pm 0.016$ & $0.464 \pm 0.058$ & $0.875 \pm 0.016$ & $0.655 \pm 0.081$ & $0.870 \pm 0.017$ & $0.473 \pm 0.057$ & $0.870 \pm 0.017$ \\
\hline GBDT4 & $0.359 \pm 0.068$ & $0.961 \pm 0.010$ & $0.247 \pm 0.049$ & $0.961 \pm 0.010$ & $0.382 \pm 0.071$ & $0.956 \pm 0.010$ & $0.260 \pm 0.050$ & 0.95 \\
\hline XGBoost4 & $0.358 \pm 0.068$ & $0.961 \pm 0.010$ & $0.247 \pm 0.049$ & $0.961 \pm 0.010$ & $0.381 \pm 0.072$ & $0.956 \pm 0.011$ & $0.259 \pm 0.050$ & $0.956 \pm 0.011$ \\
\hline BO-RF4 & $0.543 \pm 0.065$ & $0.910 \pm 0.012$ & $0.387 \pm 0.052$ & $0.910 \pm 0.012$ & $0.560 \pm 0.067$ & $0.905 \pm 0.012$ & $0.398 \pm 0.051$ & $0.905 \pm 0.012$ \\
\hline BO-GBDT4 & $0.322 \pm 0.075$ & $0.968 \pm 0.010$ & $0.220 \pm 0.052$ & $0.968 \pm 0.01$ & $0.367 \pm 0.073$ & $0.959 \pm 0.010$ & $0.244 \pm 0.051$ & $0.959 \pm 0.010$ \\
\hline BO-XGBoost4 & $0.305 \pm 0.070$ & $0.971 \pm 0.010$ & $0.208 \pm 0.050$ & $0.971 \pm 0.01$ & $0.366 \pm 0.070$ & $0.959 \pm 0.010$ & $0.241 \pm 0.049$ & $0.959 \pm 0.010$ \\
\hline \multicolumn{9}{|l|}{$\mathrm{R}_{\mathrm{s}}, \mathrm{T}_{\mathrm{s}}, \mathrm{U}_{2}$} \\
\hline RF5 & $0.638 \pm 0.079$ & $0.876 \pm 0.016$ & $0.461 \pm 0.057$ & $0.876 \pm 0.016$ & $0.651 \pm 0.083$ & $0.872 \pm 0.017$ & $0.469 \pm 0.057$ & $0.872 \pm 0.017$ \\
\hline GBDT5 & $0.315 \pm 0.049$ & $0.969 \pm 0.008$ & $0.219 \pm 0.035$ & $0.969 \pm 0.008$ & $0.335 \pm 0.053$ & $0.966 \pm 0.009$ & $0.231 \pm 0.038$ & \pm 0.009 \\
\hline XGBoost5 & $0.316 \pm 0.049$ & $0.969 \pm 0.008$ & $0.22 \pm 0.036$ & $0.969 \pm 0.008$ & $0.335 \pm 0.054$ & $0.966 \pm 0.009$ & $0.232 \pm 0.039$ & $0.966 \pm 0.009$ \\
\hline BO-RF5 & $0.527 \pm 0.061$ & $0.916 \pm 0.012$ & $0.376 \pm 0.048$ & $0.916 \pm 0.012$ & $0.541 \pm 0.065$ & $0.911 \pm 0.013$ & $0.386 \pm 0.049$ & $0.911 \pm 0.013$ \\
\hline BO-GBDT5 & $0.265 \pm 0.065$ & $0.978 \pm 0.010$ & $0.183 \pm 0.047$ & $0.978 \pm 0.010$ & $0.319 \pm 0.058$ & $0.969 \pm 0.009$ & $0.215 \pm 0.042$ & $0.969 \pm 0.009$ \\
\hline BO-XGBoost5 & $0.263 \pm 0.056$ & $0.979 \pm 0.007$ & $0.182 \pm 0.038$ & $0.979 \pm 0.007$ & $0.317 \pm 0.055$ & $0.969 \pm 0.009$ & $0.214 \pm 0.040$ & $0.969 \pm 0.009$ \\
\hline \multicolumn{9}{|l|}{$\overline{\mathrm{R}_{\mathrm{s}}}$} \\
\hline RF6 & 62 & 0.80 & $0.597 \pm 0.055$ & $0.802 \pm 0.019$ & $0.690 \pm 0.056$ & $0.807 \pm 0.025$ & $0.568 \pm 0.051$ & .025 \\
\hline GBDT6 & $0.135 \pm 0.027$ & $0.992 \pm 0.003$ & $0.107 \pm 0.021$ & $0.992 \pm 0.003$ & $0.391 \pm 0.059$ & $0.934 \pm 0.019$ & $0.286 \pm 0.039$ & 0.93 \\
\hline XGBoost6 & $0.308 \pm 0.055$ & $0.961 \pm 0.014$ & $0.236 \pm 0.041$ & $0.961 \pm 0.014$ & $0.370 \pm 0.060$ & $0.943 \pm 0.018$ & 0.042 & 0.943 \\
\hline BO-RF6 & $0.394 \pm 0.034$ & $0.938 \pm 0.011$ & $0.308 \pm 0.026$ & $0.938 \pm 0.011$ & $0.402 \pm 0.043$ & $0.934 \pm 0.015$ & $0.310 \pm 0.034$ & $0.934 \pm 0.015$ \\
\hline BO-GBDT6 & $0.294 \pm 0.119$ & $0.96 \pm 0.024$ & $0.224 \pm 0.090$ & $0.960 \pm 0.024$ & $0.392 \pm 0.071$ & $0.937 \pm 0.020$ & $0.293 \pm 0.048$ & $0.937 \pm 0.020$ \\
\hline BO-XGBoost6 & $0.349 \pm 0.064$ & $0.95 \pm 0.017$ & $0.270 \pm 0.049$ & $0.950 \pm 0.017$ & $0.366 \pm 0.064$ & $0.944 \pm 0.019$ & $0.277 \pm 0.045$ & $0.944 \pm 0.019$ \\
\hline \multicolumn{9}{|l|}{$\overline{\mathrm{T}_{\mathrm{s}}}$} \\
\hline RF7 & 0.065 & 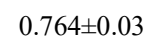 & 058 & .03 & & 32 & 0. & 032 \\
\hline GBDT7 & $0.065 \pm 0.015$ & $0.998 \pm 0.001$ & $0.049 \pm 0.012$ & $0.998 \pm 0.001$ & 090 & 0.038 & 054 & 038 \\
\hline XGBoost7 & $0.318 \pm 0.049$ & $0.959 \pm 0.013$ & $0.239 \pm 0.039$ & $0.959 \pm 0.013$ & $0.447 \pm 0.063$ & $0.918 \pm 0.021$ & $0.323 \pm 0.047$ & $0.918 \pm 0.021$ \\
\hline BO-RF7 & $0.571 \pm 0.057$ & $0.87 \pm 0.025$ & $0.449 \pm 0.056$ & $0.870 \pm 0.025$ & $0.580 \pm 0.071$ & $0.863 \pm 0.027$ & $0.454 \pm 0.067$ & $0.863 \pm 0.027$ \\
\hline BO-GBDT7 & $0.055 \pm 0.019$ & $0.999 \pm 0.001$ & $0.041 \pm 0.014$ & $0.999 \pm 0.001$ & $0.461 \pm 0.060$ & $0.913 \pm 0.024$ & $0.322 \pm 0.045$ & $0.913 \pm 0.024$ \\
\hline BO-XGBoos & $0.311 \pm 0.060$ & $0.959 \pm 0.021$ & $0.232 \pm 0.047$ & $0.959 \pm 0.021$ & $0.431 \pm 0.059$ & $0.924 \pm 0.019$ & $0.312 \pm 0.045$ & $0.924 \pm 0.019$ \\
\hline \multicolumn{9}{|l|}{$\overline{\mathrm{R}_{\mathrm{s}}, \mathrm{T}_{\mathrm{s}}}$} \\
\hline RF8 & $0.696 \pm 0.057$ & $0.809 \pm 0.021$ & $0.587 \pm 0.054$ & $0.809 \pm 0.021$ & $0.678 \pm 0.052$ & $0.813 \pm 0.025$ & .051 & 0.8 \\
\hline GBD? & $0.057 \pm 0.051$ & $0.997 \pm 0.006$ & $0.043 \pm 0.037$ & $0.997 \pm 0.006$ & & $0.966 \pm 0.013$ & 0.041 & 0.013 \\
\hline XGBoost & $0.147 \pm 0.039$ & $0.991 \pm 0.004$ & $0.108 \pm 0.027$ & $0.991 \pm 0.004$ & $0.219 \pm 0.045$ & $0.980 \pm 0.007$ & \pm 0.026 & 0.007 \\
\hline BO-RF8 & $0.393 \pm 0.036$ & $0.939 \pm 0.011$ & $0.307 \pm 0.027$ & $0.939 \pm 0.011$ & $0.401 \pm 0.044$ & $0.934 \pm 0.015$ & $0.309 \pm 0.034$ & $0.934 \pm 0.015$ \\
\hline BO-GBDT8 & $0.072 \pm 0.087$ & $0.996 \pm 0.006$ & $0.053 \pm 0.064$ & $0.996 \pm 0.006$ & $0.249 \pm 0.048$ & $0.974 \pm 0.009$ & $0.179 \pm 0.032$ & $0.974 \pm 0.009$ \\
\hline BO-XGBoost8 & $0.152 \pm 0.051$ & $0.990 \pm 0.006$ & $0.112 \pm 0.038$ & $0.990 \pm 0.006$ & $0.213 \pm 0.045$ & $0.981 \pm 0.007$ & $0.155 \pm 0.029$ & $0.981 \pm 0.007$ \\
\hline \multicolumn{9}{|l|}{$\overline{\mathrm{R}_{\mathrm{s}}, \mathrm{T}_{\mathrm{s}}, \mathrm{RH}}$} \\
\hline RF9 & $0.696 \pm 0.055$ & $0.809 \pm 0.020$ & $0.587 \pm 0.052$ & $0.809 \pm 0.020$ & $0.678 \pm 0.051$ & $0.813 \pm 0.024$ & $0.560 \pm 0.050$ & $0.813 \pm 0.024$ \\
\hline GBDT9 & $0.135 \pm 0.069$ & $0.991 \pm 0.005$ & $0.101 \pm 0.051$ & $0.991 \pm 0.005$ & $0.246 \pm 0.042$ & $0.976 \pm 0.007$ & $0.182 \pm 0.030$ & $0.976 \pm 0.007$ \\
\hline XGBoos & & & & & & & & $0.987 \pm 0.006$ \\
\hline BO-RF9 & $0.393 \pm 0.037$ & $0.939 \pm 0.011$ & $0.308 \pm 0.028$ & $0.939 \pm 0.011$ & $0.402 \pm 0.047$ & $0.934 \pm 0.016$ & $0.309 \pm 0.037$ & $0.934 \pm 0.016$ \\
\hline BO-GBDT9 & $0.054 \pm 0.059$ & $0.998 \pm 0.003$ & $0.041 \pm 0.044$ & $0.998 \pm 0.003$ & $0.200 \pm 0.047$ & $0.983 \pm 0.007$ & $0.146 \pm 0.036$ & $0.983 \pm 0.007$ \\
\hline BO-XGBoost9 & $0.102 \pm 0.029$ & $0.996 \pm 0.003$ & $0.074 \pm 0.022$ & $0.996 \pm 0.003$ & $0.167 \pm 0.037$ & $0.989 \pm 0.004$ & $0.122 \pm 0.026$ & $0.989 \pm 0.004$ \\
\hline \multicolumn{9}{|l|}{$\overline{\mathrm{R}_{\mathrm{s}}, \mathrm{T}_{\mathrm{s}}, \mathrm{U}_{2}}$} \\
\hline RF10 & $0.698 \pm 0.054$ & $0.808 \pm 0.018$ & $0.589 \pm 0.052$ & $0.808 \pm 0.018$ & $0.678 \pm 0.051$ & $0.813 \pm 0.024$ & $0.560 \pm 0.050$ & $0.813 \pm 0.024$ \\
\hline GBDT10 & $0.120 \pm 0.057$ & $0.993 \pm 0.004$ & $0.092 \pm 0.043$ & $0.993 \pm 0.004$ & $0.232 \pm 0.046$ & $0.978 \pm 0.007$ & $0.173 \pm 0.027$ & $0.978 \pm 0.007$ \\
\hline XGBoost10 & $0.101 \pm 0.018$ & $0.996 \pm 0.001$ & $0.075 \pm 0.014$ & $0.996 \pm 0.001$ & $0.168 \pm 0.030$ & $0.989 \pm 0.003$ & $0.124 \pm 0.021$ & $0.989 \pm 0.003$ \\
\hline BO-RF10 & $0.394 \pm 0.034$ & $0.938 \pm 0.011$ & $0.308 \pm 0.026$ & $0.938 \pm 0.011$ & $0.402 \pm 0.043$ & $0.934 \pm 0.015$ & $0.310 \pm 0.034$ & $0.934 \pm 0.015$ \\
\hline BO-GBDT10 & $0.060 \pm 0.066$ & $0.997 \pm 0.004$ & $0.046 \pm 0.051$ & $0.997 \pm 0.004$ & $0.193 \pm 0.041$ & $0.985 \pm 0.005$ & $0.141 \pm 0.029$ & $0.985 \pm 0.005$ \\
\hline BO-XGBoost10 & $0.082 \pm 0.025$ & $0.997 \pm 0.002$ & $0.061 \pm 0.018$ & $0.997 \pm 0.002$ & $0.158 \pm 0.032$ & $0.990 \pm 0.003$ & $0.117 \pm 0.022$ & $0.990 \pm 0.003$ \\
\hline
\end{tabular}



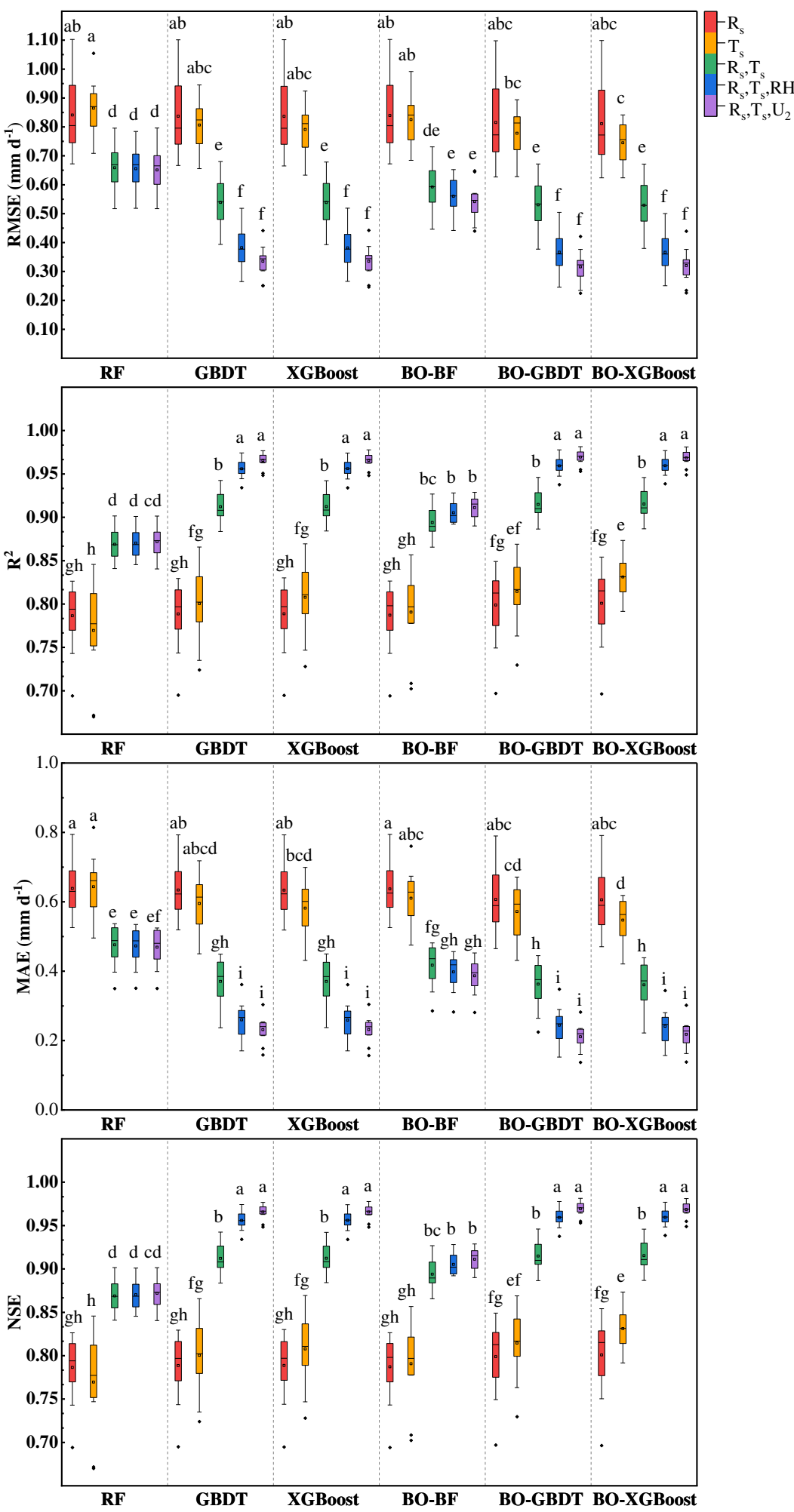

Fig. 5. Statistical performance of daily $E T_{O}$ prediction during testing under different input 

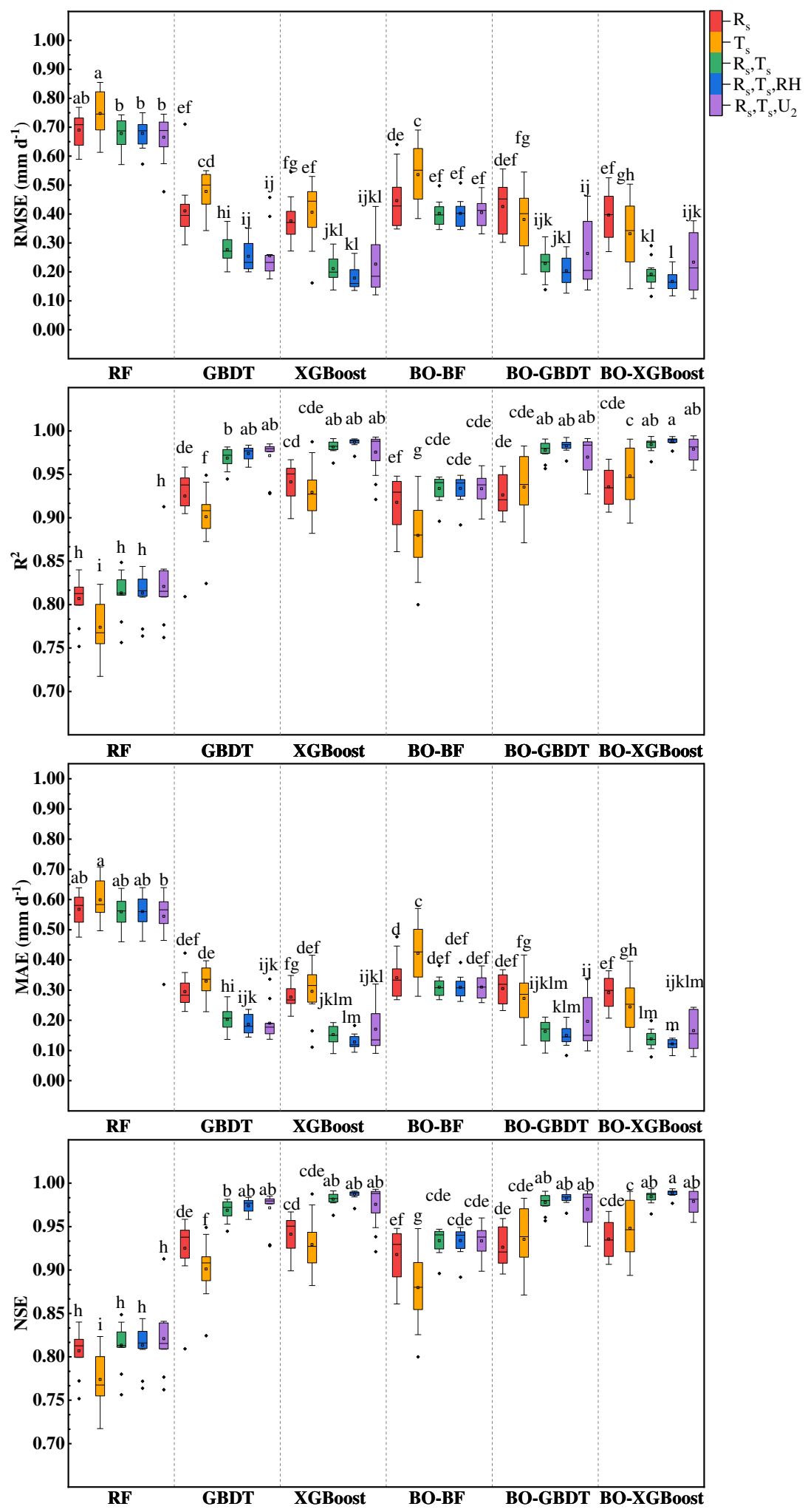

Fig. 6. Statistical performance for monthly mean $\mathrm{ET}_{\mathrm{O}}$ prediction during testing under different input combinations. 


\subsection{GPI ranking of the daily ETO and monthly mean ETO models}

Although RMSE, $\mathrm{R}^{2}$, MAE, and NSE were used, no single measure alone can be used to judge the performance of the tree-based models. Therefore, the GPI value was further calculated for all tree-based model at all stations under different input combinations (Table 5, Fig. 7, and Fig. 8). As listed in Table 5, the BO-XGBoost models, with GPI ranging from -1.954 to 1.938, 0.402 to 1.837, -0.459 to $1.982,0.436$ to 1.966 , and 0.881 to 1.993 under the input factor(s) $R_{s} ; T_{s} ; R_{s}$ and $T_{s} ; R_{s}$, $T_{s}$, and $R H$; and $R_{s}, T_{s}$, and $U_{2}$, respectively, exhibited the most effective performance at each station on the daily scale. The BO-XGBoost models also exhibited the highest GPI along with the other tree-based models on the monthly mean scale. Moreover, the hybrid tree-based models exhibited higher GPI compared with that of the standalone tree-based models at each station with different input combinations for the daily $\mathrm{ET}_{\mathrm{O}}$ and monthly mean $\mathrm{ET}_{\mathrm{O}}$; thus, the hybrid tree-based models outperformed the standalone tree-based models.

The GPI ranking comparison at each site in the study area is presented in Fig. 7 and Fig. 8. The smaller the circle radius, the larger the GPI value and the more satisfactory the model. In the comparison of daily $\mathrm{ET}_{\mathrm{O}}$ GPI value rankings, the radius of the model with $\mathrm{R}_{\mathrm{s}}$ input is larger than that with $T_{s}$ input at the Inner Mongolia site, although the opposite is true for the other sites. This indicated that the $T_{s}$ input of the Inner Mongolia site contributed more to the ETo calculation than the $R_{s}$ input, which is consistent with the results of the main factors. In the two-factor input combination, the radii of 50548 and 54311 were small, indicating that the accuracy of the model at these two sites was high; the importance of these two sites was closer to 1 than that of the other sites. When a third factor $\left(\mathrm{RH}\right.$ or $\left.\mathrm{U}_{2}\right)$ was introduced to the same site, the radius of the factor with greater importance was smaller than the factor with less importance, indicating that the model accuracy was less than the input combination with greater importance. Therefore, the importance 


\section{Table 5}

412 Values of the global performance index and rankings of the tree-based models under different input

413 combinations for daily $\mathrm{ET}_{\mathrm{O}}$ and monthly mean $\mathrm{ET}_{\mathrm{O}}$ during the testing phase.

\begin{tabular}{|c|c|c|c|c|c|c|c|c|c|c|c|}
\hline \multirow[b]{2}{*}{$\begin{array}{l}\text { Station } \\
\text { ID }\end{array}$} & \multirow[b]{2}{*}{ model } & \multicolumn{5}{|c|}{ Daily scale } & \multicolumn{5}{|c|}{ Monthly scale } \\
\hline & & $\mathbf{R}_{\mathrm{s}}$ & $\mathbf{T}_{\mathrm{s}}$ & $\mathbf{R}_{\mathrm{s}}, \mathbf{T}_{\mathrm{s}}$ & $\begin{array}{c}\mathbf{R}_{\mathrm{s}}, \mathbf{T}_{\mathrm{s}}, \\
\mathbf{R H}\end{array}$ & $\mathbf{R}_{\mathrm{s}}, \mathbf{T}_{\mathrm{s}}, \mathbf{U}_{2}$ & $\mathbf{R}_{s}$ & $\mathbf{T}_{\mathrm{s}}$ & $\mathbf{R}_{\mathrm{s}}, \mathbf{T}_{\mathrm{s}}$ & $\begin{array}{c}\mathbf{R}_{\mathrm{s}}, \mathbf{T}_{\mathrm{s}}, \\
\mathbf{R H}\end{array}$ & $\begin{array}{c}\mathbf{R}_{s}, \mathbf{T}_{\mathbf{s}}, \\
\mathbf{U}_{2}\end{array}$ \\
\hline \multirow{6}{*}{50527} & $\mathrm{RF}$ & -0.479 & 1.192 & -0.543 & -0.936 & -0.933 & -1.484 & -0.731 & -0.928 & -1.043 & -1.088 \\
\hline & GBDT & -0.467 & 1.610 & 0.876 & 1.364 & 1.423 & 0.819 & -0.348 & 0.933 & 1.477 & 1.029 \\
\hline & XGBoost & -0.449 & 1.692 & 0.866 & 1.352 & 1.411 & 0.968 & 0.906 & 1.525 & 1.737 & 1.685 \\
\hline & BO-RF & -0.479 & 1.426 & 0.311 & 0.044 & 0.038 & 1.154 & 0.177 & 0.666 & 0.616 & 0.588 \\
\hline & BO-GBDT & -0.411 & 1.687 & 0.937 & 1.455 & 1.545 & 0.818 & 0.843 & 1.345 & 1.719 & 1.501 \\
\hline & BO-XGBoost & -0.383 & 1.776 & 1.004 & 1.512 & 1.575 & 0.939 & 1.026 & 1.586 & 1.763 & 1.741 \\
\hline \multirow{6}{*}{50548} & $\mathrm{RF}$ & 1.104 & 1.041 & 0.414 & -0.186 & -0.197 & -1.778 & -0.230 & -1.847 & -1.710 & -1.825 \\
\hline & BO-RF & 1.147 & 1.542 & 1.843 & 1.863 & 1.833 & 1.115 & 0.980 & 1.749 & 1.620 & 1.639 \\
\hline & GBDT & 1.148 & 1.745 & 1.834 & 1.859 & 1.860 & 1.239 & 1.651 & 1.914 & 1.923 & 1.892 \\
\hline & BO-GBDT & 1.107 & 1.269 & 1.269 & 0.545 & 0.504 & 1.099 & 0.846 & 0.686 & 0.565 & 0.521 \\
\hline & XGBoost & 1.404 & 1.742 & 1.977 & 2.000 & 2.000 & 1.002 & 1.366 & 1.810 & 1.915 & 1.948 \\
\hline & BO-XGBoost & 1.495 & 1.837 & 1.982 & 1.966 & 1.993 & 1.211 & 1.688 & 2.000 & 2.000 & 1.973 \\
\hline \multirow{6}{*}{53391} & $\mathrm{RF}$ & -0.971 & -0.255 & -1.755 & -1.737 & -1.741 & -1.289 & -1.487 & -1.470 & -1.420 & -1.488 \\
\hline & BO-RF & -0.958 & -0.119 & -0.417 & 0.715 & 0.723 & 1.278 & 0.736 & 1.212 & 1.143 & 1.427 \\
\hline & GBDT & -0.950 & 0.371 & -0.415 & 0.712 & 0.718 & 1.507 & 1.140 & 1.562 & 1.691 & 1.624 \\
\hline & BO-GBDT & -0.969 & 0.092 & -0.983 & -0.750 & -0.847 & 1.388 & 0.246 & 0.846 & 0.746 & 0.785 \\
\hline & XGBoost & -0.829 & 0.380 & -0.382 & 0.810 & 0.739 & 1.495 & 1.079 & 1.452 & 1.422 & 1.463 \\
\hline & BO-XGBoost & -0.805 & 0.440 & -0.366 & 0.856 & 0.881 & 1.514 & 1.068 & 1.601 & 1.755 & 1.691 \\
\hline \multirow{6}{*}{53487} & $\mathrm{RF}$ & 0.067 & -0.249 & -1.445 & -1.550 & -1.588 & -1.225 & -1.736 & -1.352 & -1.376 & -1.374 \\
\hline & BO-RF & 0.100 & -0.146 & 0.027 & 1.082 & 1.245 & 1.402 & 0.726 & 1.417 & 1.525 & 1.553 \\
\hline & GBDT & 0.101 & -0.145 & 0.021 & 1.100 & 1.258 & 1.710 & 1.144 & 1.558 & 1.830 & 1.776 \\
\hline & BO-GBDT & 0.243 & 0.147 & -0.533 & -0.458 & -0.422 & 1.225 & 0.071 & 0.807 & 0.701 & 0.647 \\
\hline & XGBoost & 0.927 & 0.137 & 0.152 & 1.223 & 1.416 & 1.687 & 0.975 & 1.405 & 1.690 & 1.588 \\
\hline & BO-XGBoost & 0.943 & 0.669 & 0.083 & 1.230 & 1.322 & 1.764 & 1.236 & 1.604 & 1.897 & 1.816 \\
\hline \multirow{6}{*}{53513} & $\mathrm{RF}$ & -0.322 & 0.168 & -1.162 & -1.418 & -1.252 & -1.130 & -1.402 & -1.287 & -1.333 & -1.339 \\
\hline & BO-RF & -0.288 & 0.567 & -0.111 & 0.287 & 1.405 & 0.986 & 1.115 & 1.484 & 1.368 & 1.443 \\
\hline & GBDT & -0.282 & 0.692 & -0.122 & 0.286 & 1.399 & 1.482 & 1.400 & 1.651 & 1.660 & 1.765 \\
\hline & BO-GBDT & -0.324 & 0.347 & -0.556 & -0.682 & -0.150 & 1.232 & 0.206 & 0.716 & 0.689 & 0.662 \\
\hline & XGBoost & -0.138 & 0.674 & -0.049 & 0.402 & 1.562 & 0.903 & 1.587 & 1.545 & 1.437 & 1.776 \\
\hline & BO-XGBoost & -0.038 & 0.709 & -0.008 & 0.436 & 1.509 & 1.518 & 1.641 & 1.668 & 1.637 & 1.759 \\
\hline \multirow{6}{*}{53772} & $\mathrm{RF}$ & 0.878 & -0.288 & -0.857 & -1.184 & -0.983 & -0.932 & -1.066 & -1.195 & -1.250 & -1.172 \\
\hline & BO-RF & 0.939 & 0.385 & 0.450 & 1.179 & 1.463 & 1.812 & 1.359 & 1.548 & 1.568 & 1.579 \\
\hline & GBDT & 0.933 & 0.600 & 0.462 & 1.198 & 1.466 & 1.974 & 1.453 & 1.748 & 1.819 & 1.859 \\
\hline & BO-GBDT & 0.879 & 0.094 & -0.257 & -0.395 & 0.007 & 1.420 & 0.167 & 0.896 & 0.816 & 0.801 \\
\hline & XGBoost & 0.976 & 0.720 & 0.573 & 1.269 & 1.544 & 1.793 & 1.308 & 1.691 & 1.831 & 1.817 \\
\hline & BO-XGBoost & 0.986 & 0.788 & 0.614 & 1.275 & 1.625 & 2.000 & 1.489 & 1.716 & 1.778 & 1.921 \\
\hline \multirow{2}{*}{53798} & $\mathrm{RF}$ & 0.378 & -0.746 & -1.108 & -1.383 & -1.313 & -1.639 & -1.837 & -1.656 & -1.937 & -1.755 \\
\hline & BO-RF & 0.479 & 0.120 & 0.446 & 0.826 & 1.220 & 0.685 & 0.603 & 0.886 & 1.106 & 1.434 \\
\hline
\end{tabular}




\begin{tabular}{|c|c|c|c|c|c|c|c|c|c|c|c|}
\hline & GBDT & 0.482 & 0.278 & 0.440 & 0.829 & 1.190 & 0.771 & 0.632 & 1.331 & 1.381 & 1.618 \\
\hline & BO-GBDT & 0.384 & -0.184 & -0.173 & -0.443 & -0.248 & 0.516 & -0.697 & 0.074 & -0.063 & 0.036 \\
\hline & XGBoost & 0.579 & 0.339 & 0.545 & 1.019 & 1.354 & 0.622 & 0.544 & 1.202 & 1.249 & 1.624 \\
\hline & BO-XGBoost & 0.587 & 0.402 & 0.579 & 1.024 & 1.323 & 0.792 & 0.823 & 1.342 & 1.504 & 1.658 \\
\hline \multirow{6}{*}{53868} & $\mathrm{RF}$ & 0.706 & -0.032 & -0.512 & -0.937 & -0.886 & -1.046 & -1.117 & -1.218 & -1.281 & -1.338 \\
\hline & BO-RF & 0.808 & 0.571 & 1.099 & 1.118 & 1.520 & 1.780 & 1.225 & 1.270 & 1.650 & 1.618 \\
\hline & GBDT & 0.810 & 0.602 & 1.099 & 1.115 & 1.520 & 1.619 & 1.861 & 1.849 & 1.863 & 1.821 \\
\hline & BO-GBDT & 0.710 & 0.533 & 0.327 & -0.138 & -0.001 & 0.943 & 0.587 & 0.547 & 0.445 & 0.401 \\
\hline & XGBoost & 1.579 & 0.725 & 1.190 & 1.271 & 1.669 & 1.694 & 1.766 & 1.516 & 1.734 & 1.660 \\
\hline & BO-XGBoost & 1.739 & 1.420 & 1.224 & 1.285 & 1.676 & 1.765 & 1.969 & 1.850 & 1.885 & 1.869 \\
\hline \multirow{6}{*}{54012} & $\mathrm{RF}$ & -2.000 & 0.423 & -1.953 & -1.939 & -1.995 & -0.996 & -0.722 & -1.190 & -1.179 & -1.138 \\
\hline & BO-RF & -1.984 & 0.816 & -0.565 & 0.971 & 1.160 & 1.344 & 1.040 & 1.196 & 1.413 & 1.456 \\
\hline & GBDT & -1.989 & 0.909 & -0.549 & 0.989 & 1.147 & 1.132 & 1.362 & 1.549 & 1.633 & 1.707 \\
\hline & BP-GBDT & -2.000 & 0.630 & -1.150 & -0.765 & -0.745 & 1.041 & 0.609 & 0.568 & 0.494 & 0.496 \\
\hline & XGBoost & -1.942 & 0.891 & -0.470 & 1.035 & 1.241 & 0.922 & 1.295 & 1.481 & 1.658 & 1.642 \\
\hline & BP-XGBoost & -1.954 & 0.966 & -0.459 & 1.044 & 1.220 & 1.081 & 1.474 & 1.554 & 1.689 & 1.710 \\
\hline \multirow{6}{*}{54311} & $\mathrm{RF}$ & 1.368 & 0.348 & 0.076 & -0.439 & -0.479 & -0.919 & -0.647 & -1.107 & -1.198 & -1.162 \\
\hline & BO-RF & 1.437 & 1.057 & 1.680 & 1.538 & 1.772 & 1.313 & 1.880 & 1.731 & 1.617 & 1.711 \\
\hline & GBDT & 1.453 & 1.063 & 1.691 & 1.540 & 1.767 & 1.824 & 1.764 & 1.895 & 1.845 & 1.948 \\
\hline & BO-GBDT & 1.370 & 0.718 & 0.881 & 0.222 & 0.261 & 1.451 & 0.891 & 0.926 & 0.846 & 0.823 \\
\hline & XGBoost & 1.931 & 1.654 & 1.855 & 1.647 & 1.882 & 1.680 & 1.731 & 1.907 & 1.683 & 1.889 \\
\hline & BO-XGBoost & 1.938 & 1.453 & 1.829 & 1.648 & 1.888 & 1.800 & 1.780 & 1.921 & 1.872 & 2.000 \\
\hline \multirow{6}{*}{54449} & $\mathrm{RF}$ & 0.490 & -1.363 & -1.026 & -1.296 & -1.128 & -0.821 & -0.856 & -0.914 & $\begin{array}{l}-0.997 \\
\end{array}$ & -1.010 \\
\hline & BO-RF & 0.557 & -0.538 & 0.618 & 1.523 & 1.058 & 1.051 & 0.188 & 1.185 & 1.482 & 1.558 \\
\hline & GBDT & 0.565 & -0.467 & 0.627 & 1.537 & 1.080 & 1.159 & 1.143 & 1.738 & 1.880 & 1.864 \\
\hline & BO-GBDT & 0.489 & -0.882 & -0.049 & -0.408 & -0.324 & 1.269 & 0.726 & 0.681 & 0.703 & 0.657 \\
\hline & XGBoost & 0.700 & -0.433 & 0.623 & 1.676 & 1.170 & 1.024 & 0.840 & 1.361 & 1.772 & 1.720 \\
\hline & BO-XGBoost & 0.734 & 0.593 & 0.619 & 1.653 & 1.178 & 1.141 & 1.221 & 1.758 & 1.929 & 1.877 \\
\hline \multirow{6}{*}{54511} & $\mathrm{RF}$ & 0.856 & -2.000 & -1.472 & -1.659 & -1.376 & -0.731 & -1.199 & -0.986 & -1.068 & -1.070 \\
\hline & BO-RF & 0.899 & -0.861 & 0.057 & 1.163 & 1.259 & 1.529 & 1.210 & 1.470 & 1.477 & 1.509 \\
\hline & GBDT & 0.892 & -0.649 & 0.060 & 1.169 & 1.255 & 1.577 & 1.023 & 1.722 & 1.824 & 1.745 \\
\hline & BO-GBDT & 0.857 & -1.338 & -0.565 & -0.644 & -0.404 & 1.464 & -0.273 & 0.896 & 0.838 & 0.839 \\
\hline & XGBoost & 0.961 & -0.347 & 0.204 & 1.279 & 1.342 & 1.572 & 1.271 & 1.654 & 1.771 & 1.495 \\
\hline & BO-XGBoost & 0.942 & 0.747 & 0.263 & 1.280 & 1.410 & 1.641 & 1.292 & 1.752 & 1.847 & 1.777 \\
\hline
\end{tabular}

414 

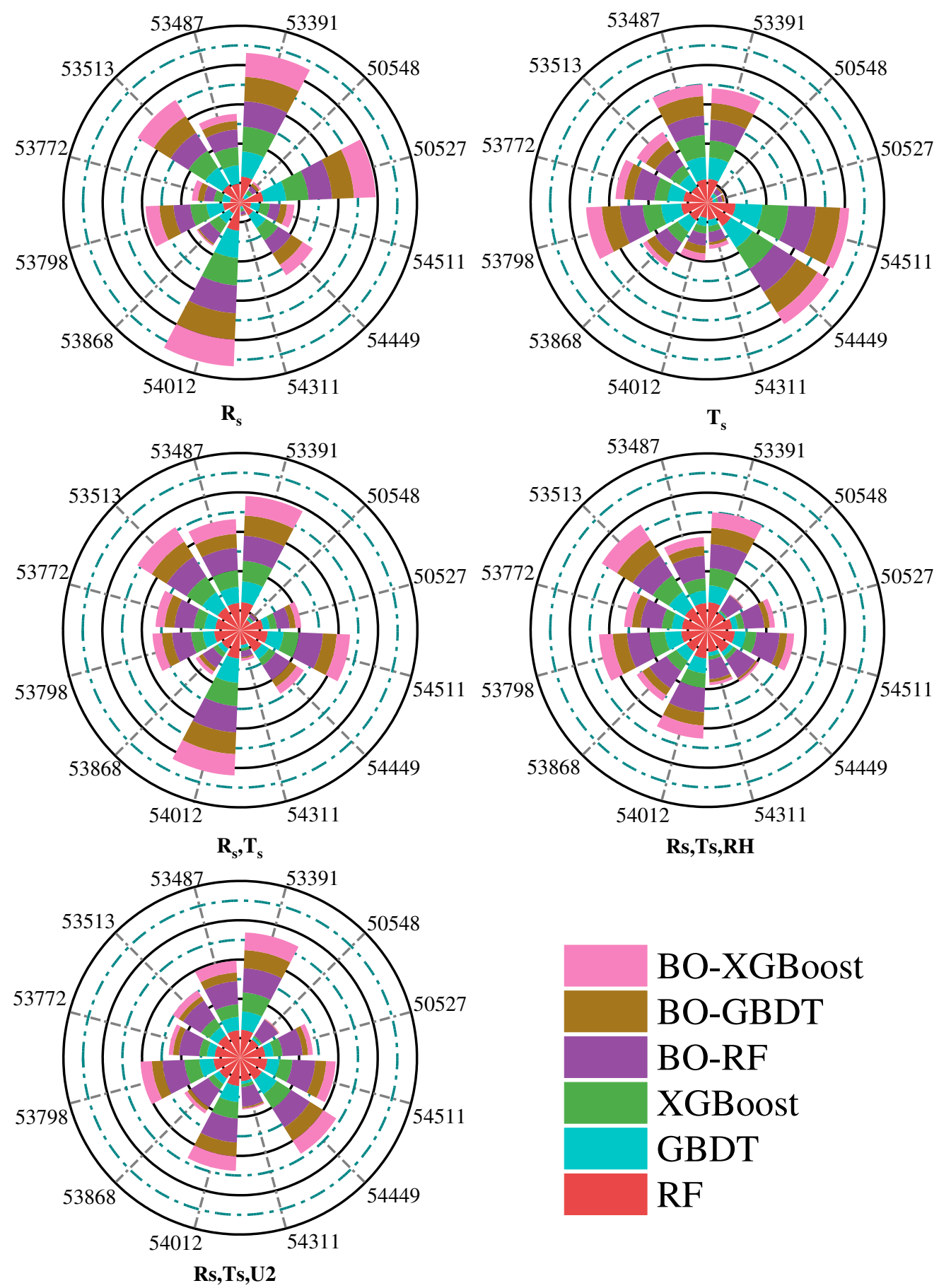

Fig. 7. GPI value of the tree-based daily $\mathrm{ET}_{\mathrm{O}}$ prediction models during the testing phase. 

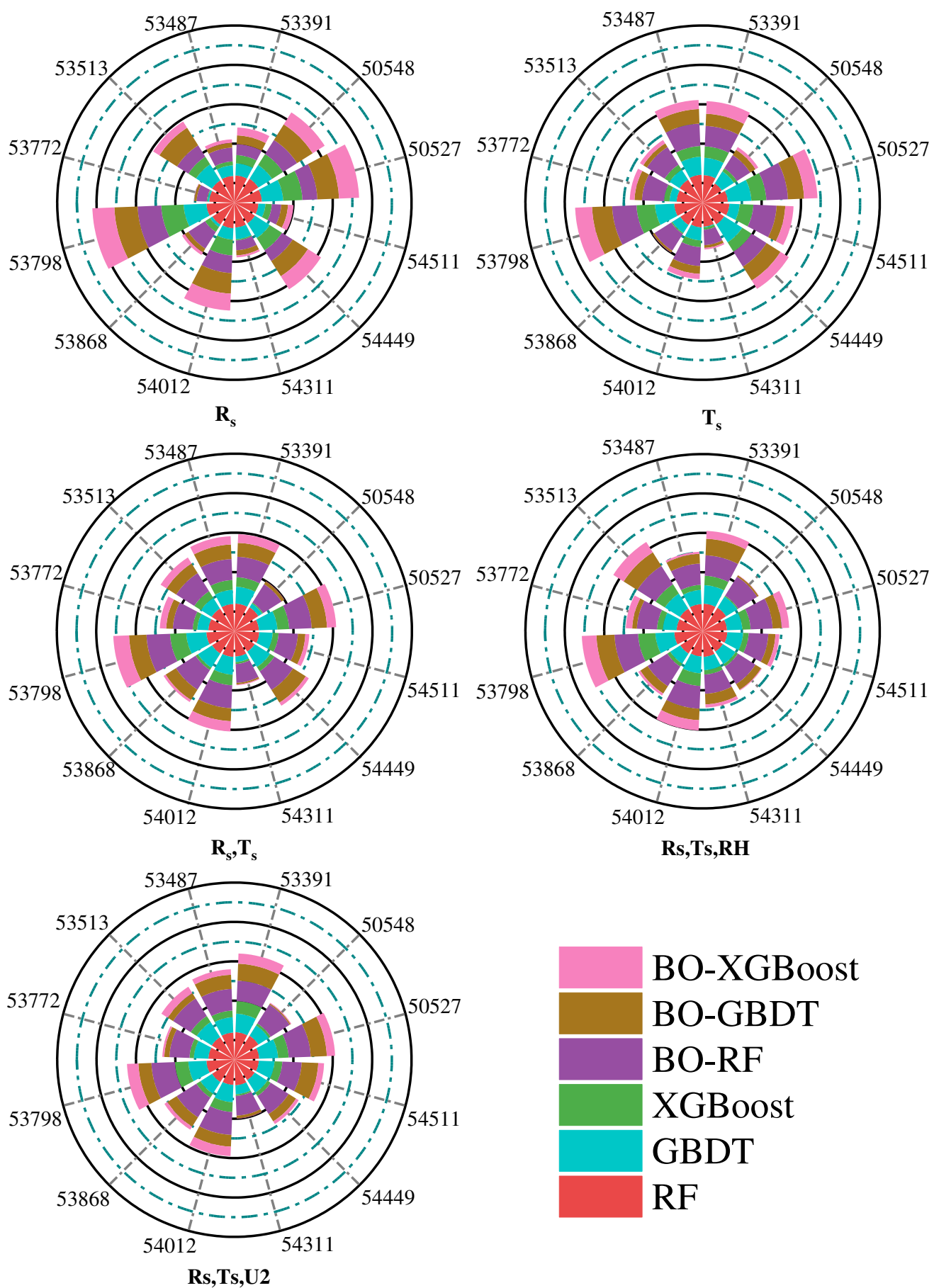

418 419

420

\section{Discussion}

phase. 


\subsection{Importance of meteorological data in daily ETo and monthly mean EToestimation}

The choice of dominant meteorological factors is critical to the prediction accuracy of machine learning $\mathrm{ET}_{\mathrm{O}}$ prediction models. To analyse the importance of meteorological data to daily $\mathrm{ET}_{\mathrm{O}}$ and monthly mean $\mathrm{ET}_{\mathrm{O}}$ prediction, three tree-based algorithms (RF, GBDT, and XGBoost) were employed. The use of tree models to select essential factors for modelling ETo can provide higher accuracy and stability with limited factors. Mohammadi et al. (2020) applied RF and four other methods for selecting the main factors of meteorological data to obtain accurate model prediction of daily ETo. The results revealed that the SVM model under RF input factors exhibited the highest performance. In the factor importance analysis of daily ETo and monthly mean ETo, $\mathrm{R}_{s}$ was determined to be the primary driving factor of daily $\mathrm{ET}_{\mathrm{O}}$ and monthly mean $\mathrm{ET}_{\mathrm{O}}$ at almost all stations and at all stations, respectively. Additionally, Chen et al. (2019) reported that $\mathrm{R}_{\mathrm{s}}$ was the key factor determining the accuracy of ETo models in hilly areas. These results indicated that $\mathrm{T}_{\mathrm{s}}$ and $\mathrm{R}_{\mathrm{s}}$ were the main factors affecting $\mathrm{ET}_{\mathrm{O}}$. For daily $\mathrm{ET}_{\mathrm{O}}$, the performance of the importance analysis of the three algorithms was similar at each station. The primary influencing factor of meteorological stations in the Inner Mongolia Autonomous Region was $\mathrm{T}_{\mathrm{s}}$ and in North China was $\mathrm{R}_{\mathrm{s}}$. Regarding the general characteristics of Inner Mongolia, $\mathrm{T}_{\mathrm{s}}$ increases sharply in spring and during the short, hot summer and then suddenly decreases in autumn, followed by long, frosty, and severely cold winters. This may explain why $\mathrm{T}_{\mathrm{s}}$ has a greater impact on daily $\mathrm{ET} \mathrm{T}_{\mathrm{O}}$ estimation. In general, $\mathrm{R}_{\mathrm{s}}$ and $\mathrm{T}_{\mathrm{s}}$ accounted for more than $90 \%$ of the contribution to daily $\mathrm{ET}_{\mathrm{O}}$ and monthly mean $\mathrm{ET}_{\mathrm{O}}$. The result of the feature importance calculation was clearly reflected in the prediction accuracy of the models.

\subsection{Prediction accuracy of the tree-based machine learning models}

To develop a more accurate model with broader application for predicting ETO, we applied 
RF, GBDT, and XGBoost to predict daily ETO and monthly mean ETO in North China based on the selected input combinations identified through factor importance analysis. On the monthly scale, the GBDT model outperformed the XGBoost model in the training phase with some input combinations but exhibited inferior performance to the XGBoost model in the testing phase. The GBDT model had an overfitting problem in which the new regression tree may have continuously fit the previous residuals, resulting in the residuals of the training set gradually approaching zero. Through a comparison of the tree-based models trained with different input combinations, we observed that the accuracy of the models predicting daily ETO and monthly mean ETO improved with the introduction of more factors. Furthermore, the XGBoost models outperformed the GBDT models in terms of R2, RMSE, MAE, NSE, and GPI, followed by the RF models. This result is consistent with that of Zhou et al. (2020), in which the XGBoost models outperformed GBDT and RF models. In this study, the daily ETO and monthly mean ETO models under different input combinations had high accuracy, demonstrating that the combination of essential factors obtained through factor importance analysis resulted in highly accurate modelling. The essential factor combination selected in the tree-based algorithm proved suitable and feasible for modelling daily ETO and monthly mean ETO.

\subsection{Comprehensive evaluation of the hybrid BO models and standalone tree-based models}

The BO algorithm was employed to identify the optimal parameter set for the relative model and to improve its accuracy (Alizamir et al., 2020; Min et al., 2021) In this study, BO was used to optimise RF, GBDT, and XGBoost, and effective results were obtained; the optimal parameter set successfully improved model accuracy and performance in the training and testing phases. Compared with the standalone tree-based models, the hybrid tree-based models (BO-RF, BOGBDT, and BO-XGBoost) had higher performance in terms of RMSE, R², MAE, NSE, and GPI, 
with BO-XGBoost outperforming the other models (Fig. 7 and Fig. 8). Alibabaei et al. (2021)

469 employed long short-term memory and bidirectional long short-term memory (BLSTM) to test ETO

470 in three locations in Portugal and applied BO to optimise the parameters of these machine learning

471 models. The results demonstrated that BLSTM optimised with BO had the highest performance,

472 which is consistent with the favourable optimisation effect reported in our study. In the training

473 and testing phases, the BO algorithm exhibited satisfactory optimisation effects on the three tree-

474 based ETo models, with the three optimised models with three-input factor combinations exhibiting

475 superior performance compared with the unoptimised models. However, when $T_{s}$ and $R_{s}$ were input, 476 the hybrid BO models also performed well in daily ETo estimation, with RMSE and MAE less 477 than $0.592 \mathrm{~mm} \mathrm{~d}^{-1}$ and $0.417 \mathrm{~mm} \mathrm{~d}^{-1}, \mathrm{R}^{2}$ and NSE more than 0.894 and 0.894 ; for the monthly 478 mean estimation, these were less than $0.402 \mathrm{~mm} \mathrm{~d}^{-1}$ and $0.310 \mathrm{~mm} \mathrm{~d}^{-1}, \mathrm{R}^{2}$ and NSE more than 4790.934 , and 0.934 , respectively, with only $\mathrm{R}_{\mathrm{s}}$. This indicated that, in the absence of large amounts 480 of data, only three factors can be used to predict monthly mean ET $_{O}$. This result clearly 481 demonstrates that the selection of essential meteorological factors based on tree-based machine 482 learning has strong rationality and reliability. Overall, the BO-XGBoost model with three factors 483 is recommended for more accurate daily $\mathrm{ET}_{\mathrm{O}}$ and monthly mean $\mathrm{ET}_{\mathrm{O}}$ prediction in North China or 484 in areas with a similar climate.

\section{5. Conclusions}

This study employed three tree-based machine learning algorithms (RF, XGBoost, and GBDT) to select essential factors for estimating daily $\mathrm{ET}_{\mathrm{O}}$ and monthly mean $\mathrm{ET}_{\mathrm{O}}$, with the performance 
489

490

491

492

493

494

(1) The result of factor importance analysis indicated that $R_{s}$ and $T_{s}$ are the main factors affecting daily $\mathrm{ET}_{\mathrm{O}}$ calculation, and $\mathrm{R}_{\mathrm{s}}$ is the main factor affecting the calculation of the monthly average $\mathrm{ET}_{\mathrm{O}}$

(2) Among the standalone tree-based models, the XGBoost model had the highest accuracy, followed by GBDT and RF. When inputting three factors $\left(\mathrm{R}_{\mathrm{s}}, \mathrm{T}_{\mathrm{s}}\right.$, and $\mathrm{U}_{2}$ or $\left.\mathrm{RH}\right)$, the accuracy of the model predicting daily $\mathrm{ET}_{\mathrm{O}}$ and monthly mean $\mathrm{ET}_{\mathrm{O}}$ was the highest in terms of $\mathrm{R}^{2}$, RMSE, MAE, NSE, and GPI, followed by models that input one and two essential factors.

(3) The BO algorithm exhibited effective optimisation on the three tree-based models. The BOXGBoost model had the highest accuracy, followed by the BO-GBDT and BO-RF models, in terms of $\mathrm{R}^{2}$, RMSE, MAE, NSE, and GPI. Under three-factor input $\left(\mathrm{R}_{\mathrm{s}}, \mathrm{T}_{\mathrm{s}}\right.$, and $\mathrm{U}_{2}$ or $\left.\mathrm{RH}\right)$, this model exhibited the highest accuracy in modelling daily $\mathrm{ET}_{\mathrm{O}}$ and monthly mean $\mathrm{ET}_{\mathrm{O}}$. Overall, $\mathrm{BO}-$ XGBoost with three-factor input is recommended as the prediction model for daily $\mathrm{ET}_{\mathrm{O}}$ and monthly mean ETo in North China.

\section{Acknowledgement}

We would like to thank the National Climatic Centre of the China Meteorological Administration for providing the climate database used in this study. This work was also supported by National Natural Science Foundation of China (51922072,51779161,51009101), Key R\&D and Promotion Projects in Henan Province (Science and Technology Development) (No. 202102110112), the Fundamental Research Funds for the Central Universities (2019CDLZ-10、 2020CDDZ-19), Experimental Technology Development Fund Project of Henan University of Science and Technology(No. SY2021008), PhD Research Startup Foundation of Henan University of Science and Technology (No. 13480025). 


\section{Author contribution}

515 Yuhang Wang; Formal analysis and investigation: Long Zhao, Xinbo Zhao, Yi Shi, Yuhang Wang, 516 Shuo Zhang; Writing - original draft preparation: Long Zhao; Writing - review and editing: Ningbo 517 Cui; Funding acquisition: Long Zhao, Ningbo Cui; Supervision: Ningbo Cui. All authors have read and agreed to the published version of the manuscript.

Data availability The datasets generated during and analysed during the current study are available from the corresponding author on reasonable request.

Code availability Not applicable.

\section{Declarations}

523 Ethics approval Not applicable.

524 Consent to participate Not applicable.

525 Consent for publication Not applicable.

526 Conflict of interest The authors declare no competing interests.

\section{References}

528 Alibabaei Khadijeh, Gaspar Pedro D., Lima Tânia M.. Modeling Soil Water Content and Reference https://doi.org/10.3390/app11115029

Armin Alipour, Jalal Yarahmadi, Maryam Mahdavi. Comparative Study of M5 Model Tree and Artificial Neural Network in Estimating Reference Evapotranspiration Using MODIS Products. Journal of Climatology, 2014, 2014: 1-11. http://dx.doi.org/10.1155/2014/839205

Babak Mohammadia, Saeid Mehdizadehb. Modeling daily reference evapotranspiration via a novel approach based on support vector regression coupled with whale optimization algorithm. Agricultural Water Management, 2020, 237. http://dx.doi.org/10.1016/j.agwat.2020.106145 
Chen T, Guestrin C. Xgboost: A scalable tree boosting system. USA: Association for Computing Machinery, 2016, 785-794. https://dl.acm.org/doi/10.1145/2939672.2939785

Haijiao Yu, Xiaohu Wen, Bo Li, Zihan Yang, Min Wu, Yaxin Ma. Uncertainty analysis of artificial intelligence modeling daily reference evapotranspiration in the northwest end of China. Computers and Electronics in Agriculture, 2020, 176: 105653. http://dx.doi.org/10.1016/j.compag.2020.105653

Ruiming Fang, Shijie Song. Daily reference evapotranspiration prediction of Tieguanyin tea plants based on mathematical morphology clustering and improved generalized regression neural network. Agricultural Water Management, 2020, 236(1): 106177. http://dx.doi.org/10.1016/j.agwat.2020.106177

Jalal Shiri. Improving the performance of the mass transfer-based reference evapotranspiration estimation approaches through a coupled wavelet-random forest methodology. Journal of Hydrology,2018, 561: 737750. https://doi.org/10.1016/j.jhydrol.2018.04.042

Jianying Yang, Qin Liu, Xurong Mei, Changrong Yan, Hui Ju, JianwenXu. Spatiotemporal Characteristics of Reference Evapotranspiration and Its Sensitivity Coefficients to Climate Factors in Huang-Huai-Hai Plain,China. Journal of Integrative Agriculture, 2013, 000(012):2280-2291. https://doi.org/ 10.1016/S2095$3119(13) 60561-4$

Jincai Zhao, Haibin Xia, Qun Yue, Zheng Wang. Spatiotemporal variation in reference evapotranspiration and its contributing climatic factors in China under future scenarios. International Journal of Climatology,2020, 40(8): 3813-3831. https://doi.org/10.1002/joc.6429

Junliang Fan, Wenjun Yue, Lifeng Wu, Fucang Zhang, Huanjie Cai, Xiukang Wang, Xianghui Lu, Youzhen Xiang. Evaluation of SVM, ELM and four tree-based ensemble models for predicting daily reference evapotranspiration using limited meteorological data in different climates of China. Agricultural and Forest Meteorology,2018, 263: 225-241. https://doi.org/10.1016/j.agrformet.2018.08.019

Lifeng Wu, Junliang Fan. Comparison of neuron-based, kernel-based, tree-based and curve-based machine learning models for predicting daily reference evapotranspiration. PloS one, 2019,14(5): 0217520. https://doi.org/10.1371/journal.pone.0217520

Lifeng Wu, Youwen Peng, Junliang Fan, Yicheng Wang. Machine learning models for the estimation of monthly mean daily reference evapotranspiration based on cross-station and synthetic data. Hydrology Research, 
Long Zhao, Xinbo Zhao, Hanmi Zhou, Xianlong Wang, Xuguang Xing Prediction model for daily reference crop evapotranspiration based on hybrid algorithm and principal components analysis in Southwest China. Computers and Electronics in Agriculture, 2021, 190: 106424. https://doi.org/10.1016/j.compag.2021.106424

LuisS. Pereira, Richard G. Allen, Martin Smith, Dirk Raes. Crop evapotranspiration estimation with FAO56: Past

Lunche Wang, Ozgur Kisi, Mohammad Zounem. Pan evaporation modeling using six different heuristic computing methods in different climates of China. Journal of Hydrology, 2017, 544: 407-427. https://doi.org/10.1016/j.jhydrol.2016.11.059

Mehdi Zamani Joharestani, Chunxiang Cao, Xiliang Ni, Barjeece Bashir, Somayeh Talebiesfandarani. PM2.5 Prediction Based on Random Forest, XGBoost, and Deep Learning Using Multisource Remote Sensing Data. Atmosphere, 2019, 10(7): 373. https://doi.org/10.3390/atmos10070373

Meysam Alizamir, Ozgur Kisi, Rana Muhammad Adnan, Alban Kuriqi. Modelling reference evapotranspiration by combining neuro-fuzzy and evolutionary strategies. Acta Geophysica, 2020, 68(3):14. https://doi.org/10.1007/s11600-020-00446-9

Mirza Junaid Ahmad, Kyung Sook Choi. Influence of climate variables on FAO Penman-Monteith reference evapotranspiration in the Upper Chenab Canal command area of Pakistan. Paddy and Water Environment, 2018, 16(3):425-438. https://doi.org/10.1007/s10333-018-0636-0

Min Yan Chia, Huang Yuk Feng, Chai Hoon Koo. Swarm-based optimization as stochastic training strategy for estimation of reference evapotranspiration using extreme learning machine. Agricultural Water Management, 2021, 243:106447. https://doi.org/10.1016/j.agwat.2020.106447

Mohammadi Babak, Mehdizadeh Saeid. Modeling daily reference evapotranspiration via a novel approach based on support vector regression coupled with whale optimization algorithm. Agricultural Water Management, 2020, 237: 106145. https://doi.org/10.1016/j.agwat.2020.106145

Muhammed A. Hassan, A. Khalil, S. Kaseb, M.A. Kassem. Exploring the potential of tree-based ensemble methods in solar radiation modeling. Applied Energy, 2017, 203: 897-916. https://doi.org/10.1016/j.apenergy.2017.06.104 Saeid Mehdizadeh, Hamid Saadatnejadgharahassanlo, Javad Behmanesh. Calibration of Hargreaves-Samani and 
Priestley-Taylor equations in estimating reference evapotranspiration in the Northwest of Iran. Archives of Agronomy and Soil Science, 2017, 63(7): 942-955. https://doi.org/10.1080/03650340.2016.1249474

Shafika Sultan Abdullah, M.A. Malek, Namiq Sultan Abdullah, Ozgur Kisi, Keem Siah Yap.Extreme Learning Machines: A new approach for prediction of reference evapotranspiration. Journal of Hydrology, 2015, 527: 184-195. https://doi.org/10.1016/j.jhydrol.2015.04.073

Srdjan Jovic, Blagoje Nedeljkovic, Zoran Golubovic, Nikola Kostic. Evolutionary algorithm for reference evapotranspiration analysis. Computers and Electronics in Agriculture, 2018, 150: 1-4. https://doi.org/10.1016/j.compag.2018.04.003

Tao Hai, Lamine Diop, Ansoumana Bodian, Koffi Djaman. Reference evapotranspiration prediction using hybridized fuzzy model with firefly algorithm: Regional case study in Burkina Faso. Agricultural Water Management, 2018, 208:140-151. https://doi.org/10.1016/j.agwat.2018.06.018

Tianao Wu, Wei Zhang, Xiyun Jiao, Weihua Guo. Comparison of five Boosting-based models for estimating daily reference evapotranspiration with limited meteorological variables. PLoS ONE, 2020, 15(6):0235324. https://doi.org/10.1371/journal.pone.0235324

Wang Lihui, He Xiaobo, Jakob F. STEINER, Zhang Dongwei, Wu Jinkui, Wang Shaoyong, Ding Yongjian. Models and measurements of seven years of evapotranspiration on a high elevation site on the Central Tibetan Plateau. Journal of Mountain Science, 2020, 17(12): 3039-3053. https://doi.org/10.1007/s11629-020$6051-1$

Xuanquan Chen, Ningbo Cui, Jiping Li. Simulation of reference crop evapotranspiration in hilly area of central Sichuan based on MARS. Transactions of the Chinese Society of Agricultural Engineering, 2019, 35(16): $152-160$.

Ye Liu, Miao Yu, Xiaoyi Ma, Xuguang Xing. Estimating models for reference evapotranspiration with core meteorological parameters via path analysis. Hydrology research, 2017, 48: 340-354. https://doi.org/10.2166/nh.2016.240

Yixiao Zhang, Zhongguo Zhao, Jianghua Zheng. CatBoost: A new approach for estimating daily reference crop evapotranspiration in arid and semi-arid regions of Northern China. Journal of Hydrology,2020, 588: 125087. https://doi.org/10.1016/j.jhydrol.2020.125087

YuFeng, Ningbo Cui, Lu Zhao, Xiaotao Hu, Daozhi Gong. Comparison of ELM, GANN, WNN and empirical 
models for estimating reference evapotranspiration in humid region of Southwest China. Journal of Hydrology, 2016, 536: 376-383. https://doi.org/10.1016/j.jhydrol.2016.02.053

623 YuFeng, Ningbo Cui, Qingwen Zhang, Lu Zhao, Daozhi Gong. Comparison of artificial intelligence and empirical models for estimation of daily diffuse solar radiation in North China Plain. International Journal of Hydrogen Energy, 2017, 42(21): 14418-14428. https://doi.org/10.1016/j.ijhydene.2017.04.084

YuFeng, Yong Peng, Ningbo Cui, Daozhi Gong, Kuandi Zhang.Modeling reference evapotranspiration using extreme learning machine and generalized regression neural network only with temperature data. Computers and Electronics in Agriculture, 2017, 136: 71-78. https://doi.org/10.1016/j.compag.2017.01.027 support vector machine for modeling daily reference evapotranspiration in a semi-arid mountain area. Hydrology Research, 2017, 48(5): 1177-1191. https://doi.org/10.2166/nh.2016.205 